\title{
Identification of fruit size associated quantitative trait loci featuring SLAF based high-density linkage map of goji berry (Lycium spp.)
}

Fazal Rehman ${ }^{1,2 \dagger}$, Haiguang Gong ${ }^{1,3 \dagger}$, Zhong Li ${ }^{4}$, Shaohua Zeng ${ }^{1,3,5}$, Tianshun Yang ${ }^{1}$, Peiyan Ai ${ }^{1,2}$, Lizhu Pan ${ }^{1}$, Hongwen Huang ${ }^{1,2^{*}}$ and Ying Wang ${ }^{1,3,5^{*}}$

\begin{abstract}
Background: Goji (Lycium spp., $2 n=24$ ) is a fruit bearing woody plant popular as a superfood for extensive medicinal and nutritional advantages. Fruit size associated attributes are important for evaluating small-fruited goji berry and plant architecture. The domestication traits are regulated quantitatively in crop plants but few studies have attempted on genomic regions corresponding to fruit traits.

Results: In this study, we established high-resolution map using specific locus amplified fragment (SLAF) sequencing for de novo SNPs detection based on $305 F_{1}$ individuals derived from $L$. chinense and $L$. barbarum and performed quantitative trait loci (QTL) analysis of fruit size related traits in goji berry. The genetic map contained 3495 SLAF markers on 12 LGs, spanning $1649.03 \mathrm{cM}$ with $0.47 \mathrm{cM}$ average interval. Female and male parents and $\mathrm{F}_{1}$ individuals' sequencing depth was 111.85 -fold and 168.72 -fold and 35.80-fold, respectively. The phenotype data were collected for 2 successive years (2018-2019); however, two-year mean data were combined in an extra year (1819). Total 117 QTLs were detected corresponding to multiple traits, of which 78 QTLs in 2 individual years and 36 QTLs in extra year. Six Promising QTLs (qFW10-6.1, qFL10-2.1, qLL10-2.1, qLD10-2.1, qLD12-4.1, qLA10-2.1) were discovered influencing fruit weight, fruit length and leaf related attributes covering an interval ranged from 27.3271.59 cM on LG10 with peak LOD of 10.48 and 14.6\% PVE. Three QTLs targeting fruit sweetness (qFS3-1, qFS5-2) and fruit firmness (qFF10-1) were also identified. Strikingly, various traits QTLs were overlapped on LG10, in particular, GFL10-2.1 was co-located with qLL10-2.1, qLD10-2.1 and qLA10-2.1 among stable QTLs, harbored tightly linked markers, while qLL10-1 was one major QTL with 14.21 highest LOD and 19.3\% variance. As LG10 harbored important traits QTLs, we might speculate that it could be hotspot region regulating fruit size and plant architectures.
\end{abstract}

\footnotetext{
* Correspondence: huanghw@scbg.ac.cn; yingwang@scib.ac.cn

${ }^{\dagger}$ Fazal Rehman and Haiguang Gong contributed equally to this work.

${ }^{1}$ Key Laboratory of South China Agricultural Plant Molecular Analysis and Genetic Improvement, Guangdong Provincial Key Laboratory of Applied Botany, South China Botanical Garden, Chinese Academy of Sciences, Guangzhou 510650, China

Full list of author information is available at the end of the article
}

(c) The Author(s). 2020 Open Access This article is licensed under a Creative Commons Attribution 4.0 International License, which permits use, sharing, adaptation, distribution and reproduction in any medium or format, as long as you give appropriate credit to the original author(s) and the source, provide a link to the Creative Commons licence, and indicate if changes were made. The images or other third party material in this article are included in the article's Creative Commons. licence, unless indicated otherwise in a credit line to the material. If material is not included in the article's Creative Commons licence and your intended use is not permitted by statutory regulation or exceeds the permitted use, you will need to obtain permission directly from the copyright holder. To view a copy of this licence, visit http://creativecommons.org/licenses/by/4.0/ The Creative Commons Public Domain Dedication waiver (http://creativecommons.org/publicdomain/zero/1.0/) applies to the data made available in this article, unless otherwise stated in a credit line to the data. 
(Continued from previous page)

Conclusions: This report highlights the extremely saturated linkage map using SLAF-seq and novel loci

contributing fruit size-related attributes in goji berry. Our results will shed light on domestication traits and further strengthen molecular and genetic underpinnings of goji berry; moreover, these findings would better facilitate to assemble the reference genome, determining potential candidate genes and marker-assisted breeding.

Keywords: Goji, SLAF-seq, Fruit size, QTL mapping, Interspecific, High-density genetic map

\section{Background}

Lycium barbarum L. and L. chinense Mill., two closely related Solanaceae species commonly known as goji, wolfberry or boxthorn. These are deciduous woody perennial shrubs, growing up to $4 \mathrm{~m}$ in height, widely distributed under warm and subtropical climate in East Asia, Southeast Asia and Europe [1-3]. It is used both in fresh and dried forms, while dry root bark and fruit also have functional importance in the medicinal industry particularly Traditional Chinese Medicine (TCM) [2]. Goji has been appreciated for its numerous health benefits as it increases the eye vision, inhibits the growth of cancer cells $[4,5]$, anti-fatigue, anti-aging, enhance metabolism and ultimately improves the immune system [1, 6]. Physiologically, the fruit of these plants contains many compounds of functional characteristics such as vitamin $\mathrm{C}$, carotenoids, flavonoids and polysaccharides [3]. Ningxia as "Daodi" region of goji production, widely renowned in China. Somehow, due to recent market demand goji cultivation areas have stretched to new regions over different climatic zones covering from $82^{\circ} \mathrm{E}$ and $115^{\circ} \mathrm{E}$ to $30^{\circ} \mathrm{N}$ and $45^{\circ} \mathrm{N}$ [7]. Particularly, cultivation of L. barbarum L. is largely extended to semi-arid climate temperate continental; Ningxia, Gansu, Inner Mongolia, Plateau continental; Qinghai and Continental arid climate; Xinjiang [8], whereas L. chinense Mill. is notably confined to temperate monsoon climate like Hubei $[7,8]$.

It is demonstrated that fruit weight controlling loci; fw2.2, fas and lc (WUSCHEL), contribute fruit size regulation by increasing carpel number, which influences yield acreage dramatically in tomato and other Solanaceae crops during domestication [9-12]. Also, fw2.2 ortholog in maize as cell number regulator gene (CNR) potentially increases heterosis vigor and yield [13]. It is witnessed that fruit size traits substantially contribute to yield components $[10,13,14]$. Previous genetic mapping and QTL studies in interspecific tomato population revealed fruit weight loci fw1.1, fw2.2, fw3.1, fw3.2 and fw11.3 each contributing more than $20 \%$ phenotypic variation. Some of these effective QTLs, fw2.2 [10], fw3.2 [15], and fw11.3 [16], responsible for fruit weight variations have already been cloned in individuals' studies. As shown, tomato fruit shape involved key QTLs, for example, Fasciated (fas) and $l c$ (WUSCHEL) two important quantitative trait loci contributed synergistically to the locule number enhancement and collectively triggered fruit size in tomato $[17,18]$. Comparatively, $L$. barbarum fruit is highly valued due to its bright red color, sweet taste and high economic returns [7]. The fruit is 2 or 3 chambered, fleshy, juicy with few or up to 15 seeds per fruit. The fruit sizes differ significantly between L. barbarum and L. chinense; ZKLC1 fruit is approximately $2.5 \mathrm{~cm}$ in size than $L$. chinense of $1.2 \mathrm{~cm}$. Current germplasm and varieties of the goji berry are diverse in their agronomic characteristics, especially oblong or narrow leaves and fruit size round to oblong [20]. We have learned from previous similar studies within the Solanaceae family that tomato [21], pepper [22], potato [23], and eggplant [12], have contributed extensively for conventional and molecular genetic research in terms of the development of molecular markers based high-density linkage maps, and mapping of qualitative and quantitative attributes [12]. Goji, a closely-related Lycium species in the Solanaceae, have been utilized for conventional genetic approaches, including hybrid development, studies on qualitative and quantitative traits [24]. Thus far, goji berry has been less explored in terms of fruit genetic aspects and breeding of quality parameters except for one recent report [25]. The accessibility of the high-density genetic map, established using genomic DNA, and single nucleotide polymorphism (SNP) markers, explore numerous opportunities to the QTL mapping, gene tagging, and isolation of candidate loci in goji berry as well as comparative mapping studies with other Solanaceae species [12, 26]. Moreover, the presence of orthologous loci or genes have already been established in several reports [12, 22, 27]. Thus, we suggest that goji berry could explain the similar trend of genetic identities or represent a novel set of candidate genes triggering fruit size for future map-based cloning. However, larger fruit sized cultivars in goji berry industry is a major breeding goal and will be a breakthrough to further marketing expansion and production increase. Molecular breeding and markerassisted selection (MAS) approaches of goji berry have not yet been attempted; moreover, it is a perennial tree plant with long duration from germination to flowering. 
Traditional breeding strategies limited due to intensive labor, cost and time inefficiency, which impeded cultivar improvement of goji berry [25].

Under rapid advancement of next-generation sequencing (NGS) technology, high-resolution genetic mapping and single nucleotide polymorphism (SNP) markers provide affordable tools for QTL mapping and MAS [28]. In the perennial fruit crops, the genetic map based on $F_{1}$ population provides a robust source to locate linkage between commercial traits and DNA markers [29]. Highly heterozygous mapping population can be successfully developed by interspecific crosses, and $F_{1}$ individuals determined under pseudo-testcross approach are widely applicable in perennial trees and forest herbs [30-32]. Recently, Gong et al. (2019) [33], constructed the first high-density genetic map of L. barbarum based on intraspecific $\mathrm{F}_{1}$ population using ddRAD-seq, which contained 23,967 SNPs with the total genetic length is $964.03 \mathrm{cM}$ and average interval is $0.040 \mathrm{cM}$. QTL analysis identified 8 significant loci targeting photosynthetic traits in 2 years dataset. Importantly, QTLs linked to net photosynthetic rate $\left(\mathrm{P}_{\mathrm{N}}\right)$ and stomatal conductance proposed critical for the plant growth and development of goji berry [33]. SLAF-seq, a high throughput reduced representation sequencing strategy for large scale SNP discovery has been practiced due to numerous advantages [34, 35], in different crop plants including pepper [36], maize [37], walnut [38], cauliflower [39], eggplant [40], and Salvia miltiorrhiza [41]. Moreover, SLAF-seq is widely applied to different woody perennial tree plants and efficiently generated genetic maps based on $F_{1}$ population [29, 33, 42]. Zhao et al. (2019) [25], reported wolfberry SNP based genetic map by SLAF-seq, which contained 6733 SNPs with the total length is $1702.45 \mathrm{cM}$ and average inter-marker distance is $0.31 \mathrm{cM}$. Moreover, QTL analysis showed 55 QTLs, of which 18 QTLs detected for fruit index on LG11 and 2 significant QTLs for leaf index on two different linkage groups based on 2 - and 3-years data, respectively [25].

Therefore, the current study executed SLAF-seq technology by establishing high-resolution linkage map utilizing $305 \quad F_{1}$ individuals of interspecific population derived from L. chinense Mill. cv. Daye and L. barbarum L. cv. ZKLC1. The map contained 3495 high-quality SLAF markers including 15,815 SNPs with a final distance of $1649.03 \mathrm{cM}$ and $0.47 \mathrm{cM}$ average marker interval. The average sequencing depth of the genetic map was 111.85 -fold in the female parent, 168.72-fold in the male parent and 35.80 -fold in $\mathrm{F}_{1}$ individuals, accounting for greater depth than previously reported [25], which ensures the accuracy of markers along the genetic map. Notably, the QTL mapping analysis identified 42 significant genomic regions or potential molecular markers closely linked with corresponding fruit size-related traits.
The high-density linkage map and promising QTLs pinpointed in this study are prerequisite for marker-assisted breeding and provide possible underpinnings for isolation of potential candidate genes underlying fruit and leaves associated commercial traits in goji berry.

\section{Results \\ Morphological traits variability analysis}

A total of 11 morphological traits, including fruit weight (FW), fruit length (FL), fruit diameter (FD), fruit shape index (FSI), number of fruits per end cluster (Nof/ec), number of fruits per node (Nof/n), number of seeds per fruit (Nos/f), 100 seed weight (100SW), leaf length (LL), leaf diameter (LD) and leaf area (LA), were evaluated based on 2 individual years (2018-2019) and extra year (1819). The analysis of variance (ANOVA) of morphological traits of 2 individual years showed significant differences $(P<0.05$ or $P<0.01)$, which indicated variation among $305 \mathrm{~F}_{1}$ progenies under different years (Table S1; Additional file 4). The descriptive statistical analysis showed coefficient of variation (CV\%) ranged between 9 to 45 for FSI and Nof/ec in 2018, and 8 to 54 for FSI, FD and Nof/ec in 2019. However, 1819 data showed that CV\% varied from 7 to 39 for FD and Nof/ec (Table S2; Additional file 5). The frequency distribution histogram and box chart among all morphological traits for individual years (2018-2019) and extra year (1819) showed normal distribution (Fig. S1a-z; Additional file 1). Normality test was performed using Kolmogorov-Smirnov (K-S) goodness of fit based on the absolute distance between cumulative distribution and values ranged from 0.02 to 0.11 , suggested positive normal distribution of all traits evaluated. The correlation analysis $(P<0.05$ or $P<$ 0.01 ) revealed extreme significant positive association detected in a few comparisons among FL, FD, 100SW, Nos/f and FW; FD, FSI, 100SW, Nos/f, LL, LD, LA and FL; 100SW, Nos/f, LD, LA and FD; LL and FSI; LD, LA and 100SW; Nof/n, LL, LD, LA and Nos/f; Nof/ec, LL and Nof/n; LD, LA and LL; LA and LD or significant positive correlation between LD and FW; LL and FD; LD, LA and FSI; Nof/ec and Nos/f; LD and Nof/n. While highly significant negative correlation was detected between FSI and FD; Nof/n and 100SW; Nof/ec and 100SW (Table 1). The extremely positive correlated traits might indicate tight association among linked markers or even candidate genes due to pleiotropic effects, such as FW and FL, FD, Nos/f, and FL and FD, FSI, LL, LD, LA. This information could help determine candidate gene prediction.

\section{Analysis of SLAF-seq data generated from $F_{1}$ population}

The sequencing data were examined to ensure the validity of the SLAF library construction. According to the goji genome size and GC content information [43], the 
Table 1 Combined correlation analysis among 11 agronomic attributes

\begin{tabular}{|c|c|c|c|c|c|c|c|c|c|c|c|}
\hline & FW & $\mathrm{FL}$ & FD & FSI & $100 \mathrm{SW}$ & Nos/f & Nof/n & Nof/ec & $\mathrm{LL}$ & LD & LA \\
\hline FW & 1 & & & & & & & & & & \\
\hline $\mathrm{FL}$ & $.657^{* *}$ & 1 & & & & & & & & & \\
\hline FD & $.804^{* *}$ & $.605^{* *}$ & 1 & & & & & & & & \\
\hline FSI & 0.051 & $.650^{* *}$ & $-.204^{* *}$ & 1 & & & & & & & \\
\hline $100 \mathrm{SW}$ & $.342^{* *}$ & $.320^{* *}$ & $.312^{* *}$ & 0.079 & 1 & & & & & & \\
\hline Nos/f & $.317^{* *}$ & $.238^{* *}$ & $.263^{* *}$ & 0.045 & 0.051 & 1 & & & & & \\
\hline Nof/n & -0.055 & -0.095 & -0.043 & -0.069 & $-.237^{* *}$ & $.163^{* *}$ & 1 & & & & \\
\hline Nof/ec & 0.109 & 0.045 & 0.074 & -0.003 & $-.152^{* *}$ & $.125^{*}$ & $.520^{* *}$ & 1 & & & \\
\hline LL & 0.099 & $.336^{* *}$ & $.145^{*}$ & $.256^{* *}$ & 0.047 & $.209^{* *}$ & $.150^{* *}$ & 0.112 & 1 & & \\
\hline LD & $.144^{*}$ & $.297^{* *}$ & $.228^{* *}$ & $.134^{*}$ & $.172^{* *}$ & $.276^{* *}$ & $.122^{*}$ & -0.006 & $.825^{* *}$ & 1 & \\
\hline LA & $.156^{* *}$ & $.261^{* *}$ & $.174^{* *}$ & $.133^{*}$ & $.200^{* *}$ & $.262^{* *}$ & 0.077 & 0.004 & $.821^{* *}$ & $.887^{* *}$ & 1 \\
\hline
\end{tabular}

** Correlation is significant at the 0.01 level (2-tailed)

* Correlation is significant at the 0.05 level (2-tailed)

FW fruit weight, FL fruit length, FD fruit diameter, FSI fruit shape index, Nof/ec number of fruits/end cluster, Nof/n number of fruits/node, Nos/f number of seeds/ fruit, 100SW 100 seed weight, LL leaf length, LD leaf diameter, LA leaf area

pepper genome was selected as the reference genome utilizing self-developed predicted software and established protocol [44]. For optimal enzyme digestion the following guidelines were practiced such as; 1 ) lowest presence of selected digested fragments in the repetitive sequences, 2) regular distribution of digested fragments in the reference genome, 3 ) length and specificity of the digested fragment and specific pilot experiment system must comply uniformity, 4) the final number of digested fragments or SLAF tags must meet the expected number of tags [44, 45]. RsaI and HinCII were used for the SLAF library construction and 221,608 SLAF tags were obtained based on the pre-SLAF experiment.

The cross-check revealed normality of the SLAF library with paired-end mapping reads of $92.16 \%$ and enzymatic digestion efficiency of $93.11 \%$. A total of $3021.32 \mathrm{M}$ clean reads of data with a length of $200 \mathrm{bp}$ were generated for goji berry, comprising $37 \mathrm{M}, 30 \mathrm{M}$ and $9 \mathrm{M}$ total paired-end reads in the female, male parents and offspring, respectively. The source data quality was ensured by average Q30 ratio of 95.04 and 39.62\% GC. Correspondingly, in the female and male parent total number of SLAFs developed were 155,002 and 217, 485 with an average depth of 72.43 -fold and 60.43-fold of each SLAF marker, while for $305 F_{1}$ individuals 199, 560 SLAFs were generated, with an average depth of 15.23-fold (Table 2). Of 494,472 high-quality SLAFs, 214,961 were polymorphic with $43.47 \%$ of polymorphism rate; furthermore, 279,356 non-polymorphic and 155 repetitive SLAFs were identified. After filtering out polymorphic SLAFs, a total of 40,616 SLAFs was classified into eight segregation patterns (Fig. 1). For the $F_{1}$ crosspollinated population, only 7 segregation patterns $(\mathrm{ab} \times \mathrm{cd}$, ef $\times$ eg, hk $\times$ hk, lm $\times \mathrm{ll}, \mathrm{nn} \times \mathrm{np}, \mathrm{ab} \times \mathrm{cc}, \mathrm{cc} \times \mathrm{ab})$ were retained for genetic mapping. We followed the genotyping quality criteria to filter out low quality and redundant markers using salient steps of filtering process (See "Methods" section). Collectively, we obtained 5669 total SLAFs out of which only 3495 high-quality SLAF markers were eventually utilized to construct an integrated (combined female and male parent map) high-density genetic map of goji berry with an average parental sequencing depth of more than 100-fold and offspring > 10-fold (Table 3). Among 3495 SLAF markers, 'SNP_only' were pre-dominant with $61.08 \%$ on the integrated map followed by 'InDel_only' $(0.59 \%)$ and SNP \& InDel (38.55\%) (Fig. 2).

\section{Basic characteristics of the genetic map}

After performing fine filtering process out of 5669 SLAFs only 3495 high-quality SLAF markers that met the quality standard were mapped onto the integrated

Table 2 Data description of SLAF-library Sequencing

\begin{tabular}{lllllll}
\hline Samples & Total Reads & Q30 (\%) & GC (\%) & SLAFs & Total Depth & Average Depth \\
\hline Female parent & $37,527,272$ & 94.86 & 40.69 & 155,002 & $11,227,106$ & 72.43 \\
Male parent & $30,768,211$ & 94.86 & 39.49 & 217,485 & $13,143,686$ & 60.43 \\
Offspring & $9,682,038$ & 95.04 & 39.62 & 199,560 & $3,038,879$ & 15.23 \\
Control & $2,233,676$ & 95.16 & 40.51 & - & - & - \\
Total & $3021,316,939$ & 95.04 & 39.62 & - & - & - \\
\hline
\end{tabular}




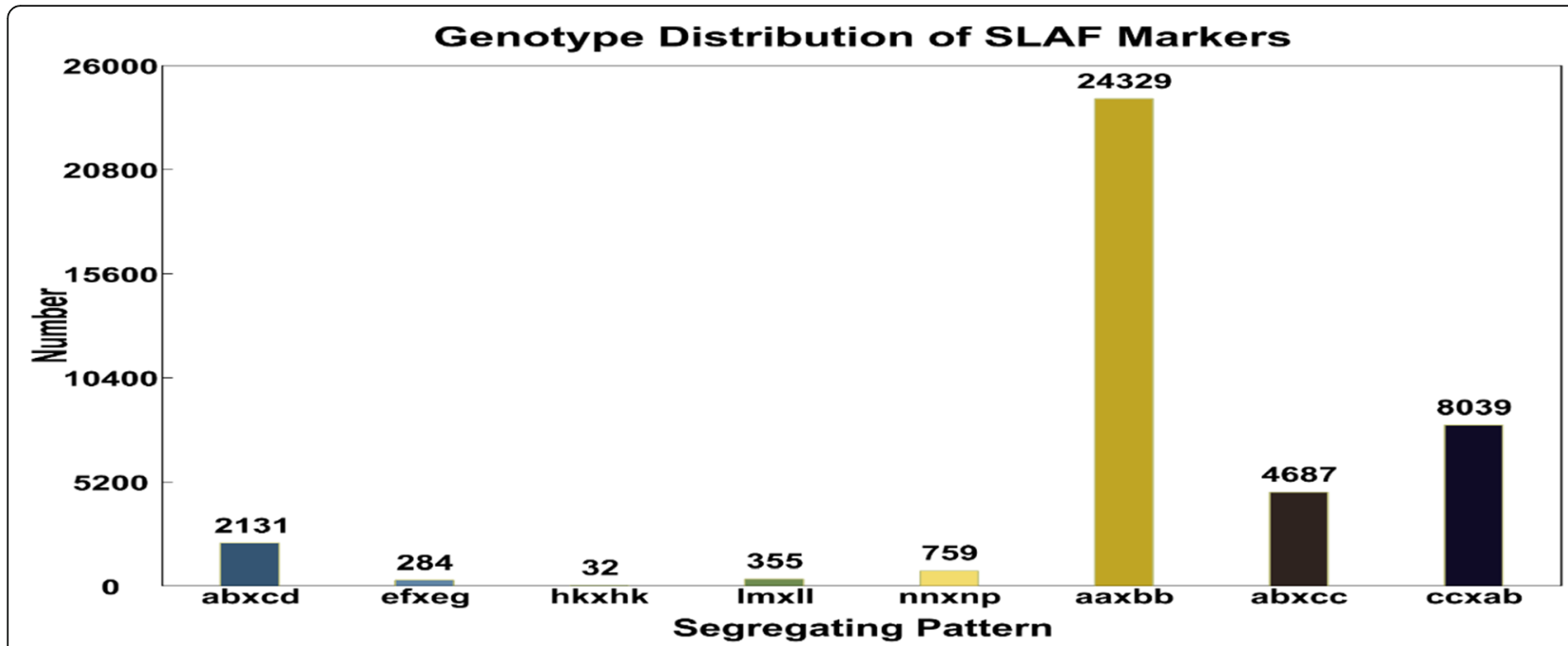

Fig. 1 Segregation pattern of polymorphic SLAF markers for $F_{1}$ interspecific population. ${ }^{*}$ The $x$-axis depicts segregation pattern and $y$-axis shows the number of markers

genetic map comprised of $522 \mathrm{~L}$. barbarum (male) and 3143 L. chinense (female) markers with a ratio of $61.65 \%$. (Fig. 3, Table 4). The average coverage of SLAF markers on the integrated genetic map was $111.82 \mathrm{cM}$ for female and $168.72 \mathrm{cM}$ male parent and $35.80 \mathrm{cM}$ each $F_{1}$ individual, respectively. The final map was $1649.03 \mathrm{cM}$ in length, confined to 12 LGs equal to the gametic chromosome number of $L$. barbarum with an average interval of $0.47 \mathrm{cM}$.

The largest and the most saturated linkage group was LG10, harbored 903 SLAF markers covering a length of $227.25 \mathrm{cM}$ with the least average interval of $0.25 \mathrm{cM}$, while the smallest LG11 contained 36 markers with $19.16 \mathrm{cM}$ genetic distance and $0.55 \mathrm{cM}$ average interval.

Table 3 Detail Summary of high-quality SLAFs and SLAF markers

\begin{tabular}{ll}
\hline High-Quality SLAFs & \\
\hline Number of *SLAFs & 494,472 \\
Average depth in female parent & 72.43 \\
Average depth in male parent & 60.43 \\
Average depth in individuals & 15.23 \\
Polymorphic *SLAFs & \\
Number of polymorphic *SLAFs & $214,961(43.47 \%)$ \\
Number of non-polymorphic *SLAFs & $279,356(56.50 \%)$ \\
Number of repetitive *SLAFs & $155(0.03 \%)$ \\
High-Quality *SLAF markers & \\
Number of *SLAF markers & 3495 \\
Average depth in Female parent & 111.85 \\
Average depth in male parent & 168.72 \\
Average depth in individuals & 35.80 \\
\hline
\end{tabular}

*SLAF- Specific locus amplified fragment
Surprisingly, the parental map largest linkage group was the same as that of the integrated map (LG10), harbored 142 markers spanning $147.16 \mathrm{cM}$ for the male and 811 markers covering $292.32 \mathrm{cM}$ for the female maps. However, LG8 and LG11 were short with an equal number of markers covering $20.47 \mathrm{cM}$ and $2.15 \mathrm{cM}$ genetic distances for the male map, respectively. In the same manner, LG9 belong to the female map was the smallest with $61.30 \mathrm{cM}$ genetic distance (Table 4). The map uniformity revealed based on maximum gap and average gap <=5, which was experienced in LG1 with $16.99 \mathrm{cM}$ and $99.10 \%$, respectively. Moreover, we detected 15,810 SNPs ranges between 163 (LG11) to 4013 SNPs (LG10) on the integrated map along with 6135 transversions and 9675 transitions (Table 4). Gong et al. (2019) [25], constructed the integrated genetic map based on intraspecific population with 23,967 SNP markers, spanned $964.03 \mathrm{cM}$ of final genetic distance and $0.040 \mathrm{cM}$ average marker interval, which reflected highly saturated map [33]. Similarly, another study reported wolfberry highdensity SNP based genetic map with a final distance of $1702.45 \mathrm{cM}$ and $0.31 \mathrm{cM}$ average interval [25]. In comparison with previous reports [25, 33], the current study revealed interspecific genetic map of goji berry with final genetic coverage of $1649.03 \mathrm{cM}$ and $0.47 \mathrm{cM}$ mean marker interval. In particular, LG6 \& LG10 was observed with least inter marker distances indicated maximum saturation and might be considered recombination hotspots in this population.

\section{Assessment of high-density genetic map of goji berry}

To evaluate the quality of the genetic map, we performed several mapping approaches; Firstly, the integrity of all mapped markers was carried out between $305 \mathrm{~F}_{1}$ 


\section{SNP-only' SLAF 口 InDel-only' SLAF $\square$ SNP\&InDel' SLAF}

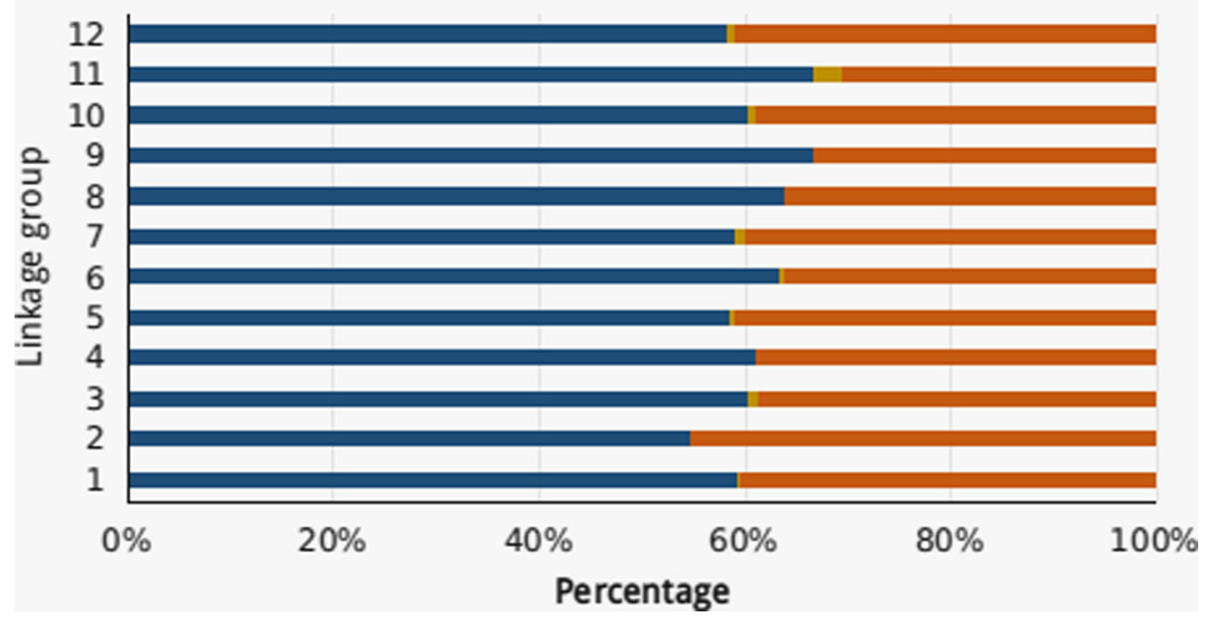

Fig. 2 Percentages of various types of markers detected among each linkage group. *The $x$-axis indicates the percentage of three types of markers: "SNP_only, InDel_only, and SNP_InDel on each linkage group, while the $y$-axis indicates the 12 linkage groups of the integrated map

individuals to ensure the quality of the genotyping, accounting for $99.03 \%$ on average (Fig. S2; Additional file 1). Secondly, haplotype mapping was performed among each individual and both parents using 3495 SLAFs to locate genotyping errors (Fig. S3a-l; Additional file 2). In the current study, the average percentage of the missing fragments were $0.0125 \%$, represent the true quality of the map. It was verified that the interspecific $F_{1}$ population was highly purified and suitable for high-density genetic map construction. DNA markers located on each linkage group were dispensed regularly with an average interval of $0.47 \mathrm{cM}$ between adjacent markers despite high-rate of recombination events in $F_{1}$ individuals. Thirdly, we displayed heatmaps to show the recombination frequencies among markers located on each linkage group to further evaluate the quality of the genetic map using pairwise recombination rate. The heatmaps depicted markers on the map were ordered accurately and pair-wise recombination rates were considerably low among adjacent markers and represented with yellow color diagonally. The purple color, particularly more visible in LG6 and LG10, reflected the highest recombination rates among adjacent markers (Fig. S4a-l; Additional file 2). Finally, 18 markers with segregation distortion $(P<0.001)$ were integrated in the construction of the genetic map, accounting for $0.34 \%$ of all mapped markers.

\section{QTL mapping analysis}

By using the high-density genetic map of goji berry, we plotted a large number of QTLs covering 13 traits. QTL analysis was performed using the composite interval mapping model with MapQTL v. 6.0 estimating 11 agronomical important traits for 2 individual years (20182019) and extra year (1819). Moreover, fruit quality traits; fruit sweetness (soluble solid content ${ }^{\circ}$ brix) FS (SSC) and fruit firmness (FF) traits were examined during summer season, 2019. In order to set the LOD threshold, we performed 1000 permutation test and the threshold value was used to evaluate the statistical significance of each QTL. In this way, significant QTLs corresponding to particular traits was identified in 2 individual years and extra year consistently. As a result, most of the QTLs including promising QTLs relating to Nos/f, FD, FW, FL and leaf related traits were positioned in linkage group 10 (Fig. 4, Table 5). Other traits QTLs such as FSI, Nof/ec, Nof/n, 100SW, FS (SSC) \& FF were located in different linkage groups (Fig. S5, S6, S7). In addition, $q F S-1, q F S-2$ corresponding to fruit sweetness with maximum phenotypic variance of $9.2 \%$ and $q F F-1$ to fruit firmness with $8.1 \%$ of PVE were mapped on LG3, 5 and 10, respectively (Fig. 4 and S6, Table 5). Collectively, 117 QTLs targeting 13 important traits were found (Table 5 and S3; Additional file 6). Of which 78 QTLs in 2 individual years including 6 stable QTLs and 36 QTLs in extra year (1819) and only 3 QTLs in 2019 was detected (Table 5 and S3; Additional file 6). The number of markers located in corresponding QTLs ranged between 1 to 105 , explaining highest PVE up to 19.3\% with 14.21 Logarithm of Odds (LOD) covering all QTLs (Table 5 and S3; Additional file 6). QTLs detected corresponding to each trait in 2 individual years were described as follows:

Fruit weight ( $f w$ ): Total 9 fruit weight QTLs were detected on LG10 and 6, of which major QTL was qFW10-1 with explained phenotypic variance of $11.1 \%$. One stable QTL ( $q F W 10-6.1$ ) was located on LG10 with genetic distance from 65.89 to $71.86 \mathrm{cM}$ and peak 

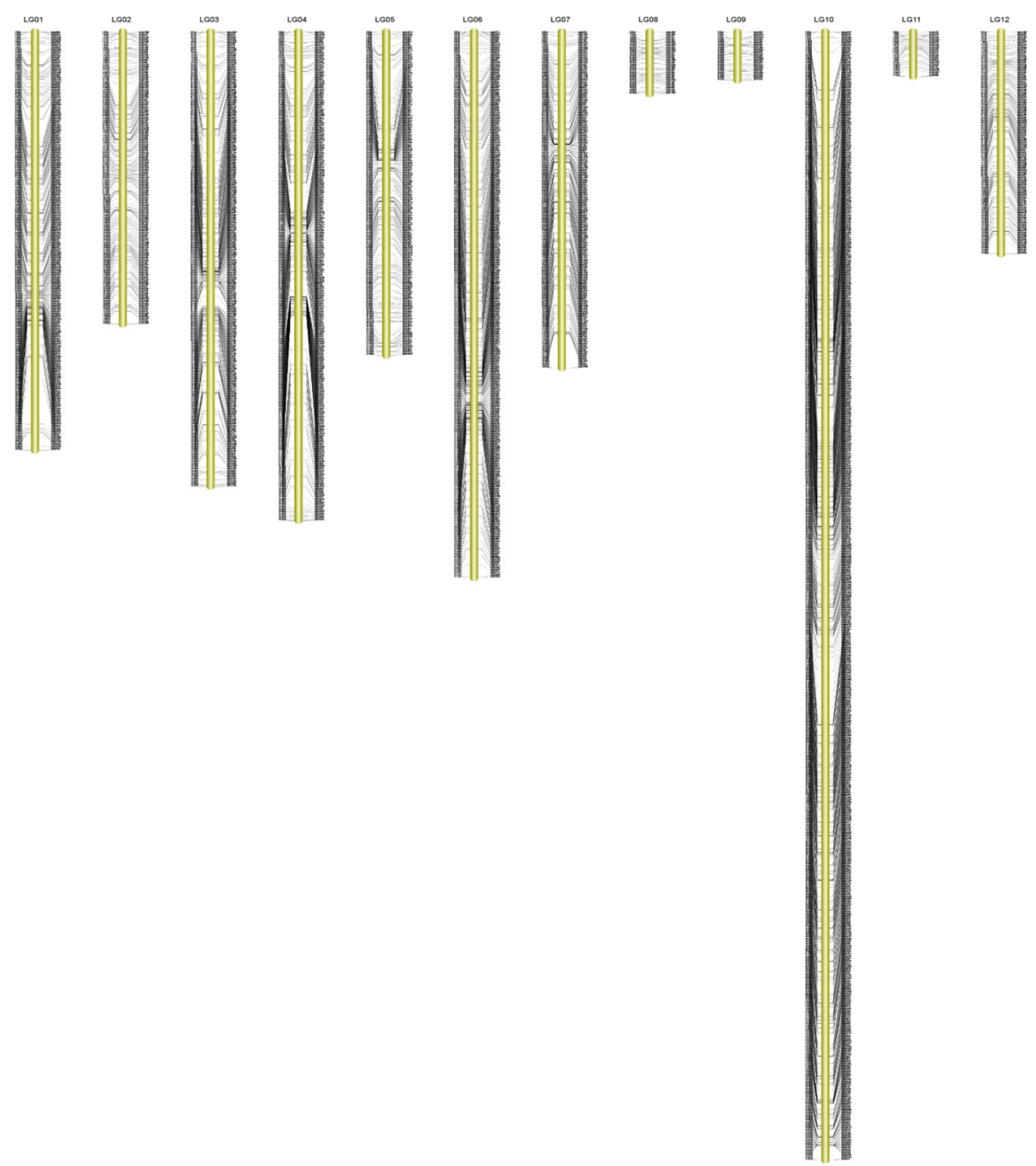

Fig. 3 The high-density genetic map presents the distribution of SNP markers on 12 linkage groups (LGS) of goji berry (Lycium Spp.). *The black bars on each linkage group show markers distribution

LOD value up to 6.01 along with corresponding PVE of 8.7\% (Fig. 4). Moreover, 4 QTLs controlling FW was mapped on LG10 under extra year (1819) and qFW102 was confirmed as effective loci with maximum of 11.6\% PVE (Table 5 and S3, additional file 6).

Fruit length $(f l): 5$ QTLs responsible for fruit length were found on LG10. The maximum phenotypic variance of $11.5 \%$, corresponding to $q F L 10-1$ considered as significant QTL. qFL10-2.1 targeting fruit length was observed as stable QTL on LG10, spanned an interval ranged from 44.64-56.88 cM with corresponding highest LOD of 6.54 and $9.5 \%$

phenotypic variance (Fig. 4). Another 4 FL QTLs were detected on LG10 in 1819 with highest PVE of 12.4\% corresponding to qFL10-1 (Table 5 and S3, additional file 6).
Fruit diameter $(f d)$ : Only 5 QTLs corresponding to fruit diameter were located on LG10, spanned an interval ranged from 4.14-71.36 cM with a LOD value up to 7.8 and $11.1 \%$ PVE. $q F D 10-1$ was detected as a major QTL for controlling fruit diameter with maximum phenotypic variance of $11.1 \%$ (Table S3, additional file 6). While 3 QTLs targeting FD were also found on LG10 with highest $12.8 \%$ PVE for $q F D 10-1$ in 1819 (Table 5).

Fruit shape index $(f s i): 8$ QTLs controlling fruit shape index was observed on LG1, 2, 3, 7, 8 and 11, covered genetic interval ranged from $0.85-124.76 \mathrm{cM}$ with LOD between 4.55 to 7.08 and explained variance of 6.6$10.2 \%$. The significant QTL controlling fruit shape index was qFSI8-4 with maximum PVE of 10.2\% (Table S3, additional file 6). In addition, 3 QTLs 
Table 4 Basic Characteristics of High-density genetic map of goji berry

\begin{tabular}{|c|c|c|c|c|c|c|c|c|c|c|c|c|c|c|}
\hline \multirow[b]{2}{*}{ *LGsID } & \multicolumn{3}{|c|}{ Number of markers } & \multicolumn{3}{|c|}{ Distance (cM) } & \multicolumn{3}{|c|}{ Average distance (cM) } & \multicolumn{2}{|c|}{ Integrated map } & \multicolumn{3}{|l|}{ SNPS } \\
\hline & ${ }^{*} \mathrm{Fm}$ & ${ }^{*} \mathrm{Mm}$ & ${ }^{*} \operatorname{lm}$ & ${ }^{*} \mathrm{Fm}$ & ${ }^{*} \mathrm{Mm}$ & ${ }^{*} \mid \mathrm{m}$ & ${ }^{*} \mathrm{Fm}$ & ${ }^{*} \mathrm{Mm}$ & ${ }^{*} \mathrm{Im}$ & Gaps $<=5 \%$ & *Max. Gap & *SNPS & ${ }^{*} \operatorname{Trv}$ & ${ }^{*}$ Tri \\
\hline $\mathrm{LG1}$ & 269 & 79 & 335 & 170.32 & 127.48 & 159.2 & 0.64 & 1.63 & 0.48 & 99.1 & 16.99 & 1541 & 575 & 966 \\
\hline LG2 & 220 & 29 & 234 & 210.97 & 144.49 & 184.2 & 0.96 & 5.16 & 0.79 & 99.14 & 8.85 & 1044 & 411 & 633 \\
\hline LG3 & 335 & 49 & 364 & 181.81 & 139.5 & 162.4 & 0.54 & 2.91 & 0.45 & 100 & 4.64 & 1664 & 640 & 1024 \\
\hline LG4 & 373 & 30 & 391 & 170.51 & 111.96 & 158.91 & 0.46 & 3.86 & 0.41 & 99.23 & 11.2 & 1874 & 748 & 1126 \\
\hline LG5 & 228 & 45 & 259 & 125.24 & 103.94 & 115.74 & 0.55 & 2.36 & 0.45 & 100 & 4.94 & 1190 & 442 & 748 \\
\hline LG6 & 411 & 43 & 437 & 204 & 111.81 & 161.19 & 0.5 & 2.66 & 0.37 & 100 & 3.8 & 2028 & 773 & 1255 \\
\hline LG7 & 237 & 42 & 269 & 144.18 & 134.76 & 149.38 & 0.61 & 3.29 & 0.56 & 99.63 & 15.07 & 1195 & 476 & 719 \\
\hline LG8 & 46 & 6 & 50 & 87.06 & 20.47 & 83.31 & 1.93 & 4.09 & 1.7 & 91.84 & 6.2 & 219 & 89 & 130 \\
\hline LG9 & 31 & 11 & 39 & 61.3 & 37.23 & 55.09 & 2.04 & 3.72 & 1.45 & 94.74 & 7.01 & 170 & 64 & 106 \\
\hline LG10 & 811 & 142 & 903 & 292.32 & 147.16 & 227.25 & 0.36 & 1.04 & 0.25 & 99.89 & 7.62 & 4013 & 1581 & 2432 \\
\hline LG11 & 36 & 6 & 36 & 34.65 & 2.15 & 19.16 & 0.99 & 0.43 & 0.55 & 100 & 2.55 & 163 & 56 & 107 \\
\hline LG12 & 146 & 40 & 178 & 226.59 & 97.82 & 173.2 & 1.56 & 2.51 & 0.98 & 98.31 & 9.39 & 709 & 280 & 429 \\
\hline Total & 3143 & 522 & 3495 & 1908.95 & 1178.77 & 1649.03 & 0.61 & 2.31 & 0.47 & 98.49 & 16.99 & 15,810 & 6135 & 9675 \\
\hline
\end{tabular}

${ }^{*}$ Fm Female map, Mm Male map, Im Integrated map, Max maximum, SNPs Single nucleotide polymorphism, Trv Transversions, Tri Transitions, LG linkage group

corresponding to FSI were detected on LG7 and 8 in extra year. The major QTL was $q F S I 8-3$ with highest phenotypic variance of $8.5 \%$ (Table 5 ).

Number of fruits per end cluster (nof/ec): QTL analysis identified 8 QTLs targeting number of fruits per end cluster were positioned on LG5, 6, 8 and 9, spanned an interval ranged from 9.61-144.52 cM with LOD between 3 to 4.91 and 4.4 to $7.2 \%$ PVE. qNof/ec8-3 was detected as significant QTL with larger PVE of 7.2\% (Table S3, additional file 6). In 1819 only 1 QTL was detected on LG5 with 8.1\% PVE for Nof/ec (Table 5). Number of fruits per node (nof/n): Total 7 QTLs corresponding to number of fruits per node were located on LG5 and 9, spanned an interval ranged from 14.05-92.48 with LOD value between 6.48 to 7.11 and
9.3 to $10.2 \%$ PVE. We detected $q$ Nof $/ n 5-6$ as major QTL with maximum $10.2 \%$ variance (Table S3, additional file 6). Only 2 QTLs targeting Nof/n were mapped on LG5 and 9 with extreme variation of $12.5 \%$ for $q N o f / n 5-1$ in extra year (1819) (Table 5).

Number of seeds per fruit (nos/f): We detected 4 QTLs on LG10, covered an interval ranged from 32.09-51.33 cM with LOD between 4.45 to 7.72 and PVE of 6.5 to $11 \%$. Two major QTLs (qNos/f10-2, qNos/f10-3) were observed with the highest variance of $11 \%$ (Table S3, additional file 6). Another $3 \mathrm{Nos} / \mathrm{f}$ QTLs were identified on LG10 with larger PVE of $11.6 \%$ for qNos/f10-1 in 1819 (Table 5).

100 seed weight (100sw): 3 QTLs targeting 100 seed weight were detected on LG7, 9 and 12. These QTLs

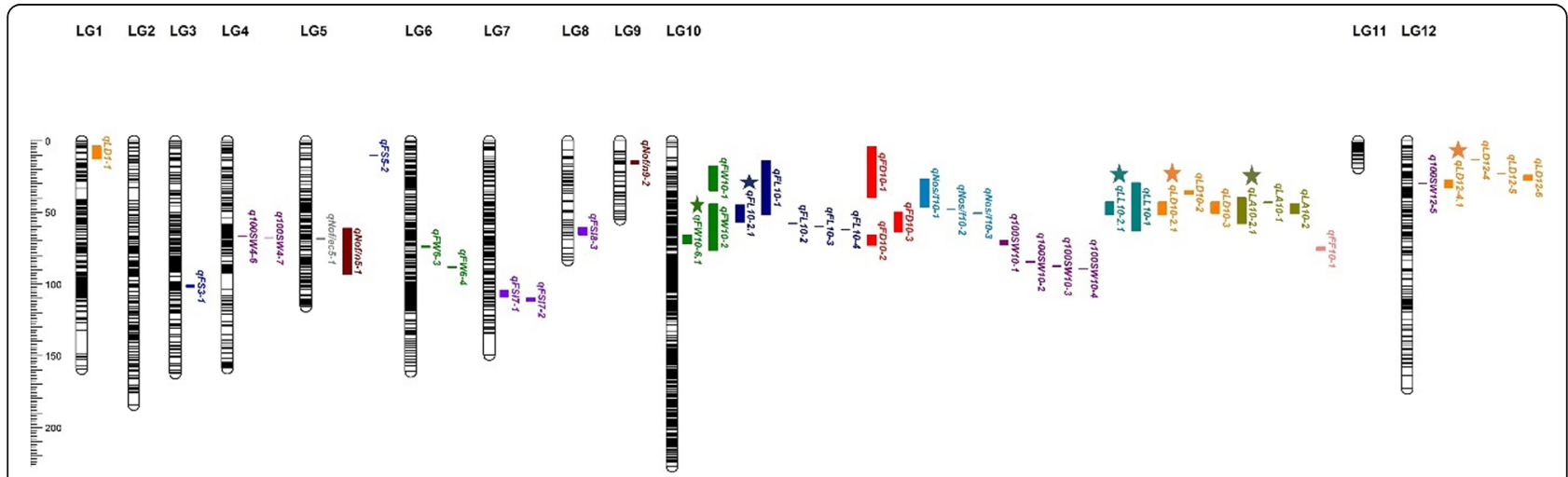

Fig. 4 The high-density genetic map of goji berry and stable QTLs mapping on linkage groups detected under 2 individual year and extra year. *Different vertical color tiles represent various traits QTLs. Green, QFW_fruit weight; dark blue, gFL_fruit length; red, qFD_fruit diameter; blue violet, qFSI_fruit shape index; grey, qNof/ec_number of fruits per end cluster; brown, qNof/n_number of fruits per node; steel blue, qNos/f_number of seeds per fruit; dark magenta, q100SW_100 seed weight; teal, qLL_leaf length; orange, qLD_leaf diameter; olive, qLA_leaf area, blue, qFS_fruit sweetness, salmon, qFF_fruit firmness. Stars indicate stable QTLs identified in 2 individual years consistently 
Table 5 Promising QTLs detail information detected under 2 individual years and extra year (1819) data

\begin{tabular}{|c|c|c|c|c|c|c|c|c|}
\hline \multirow[t]{2}{*}{ Traits } & \multirow[t]{2}{*}{ Year } & \multirow[t]{2}{*}{ QTLS } & \multirow[t]{2}{*}{ LGs } & \multicolumn{3}{|c|}{ Interval (cM) } & \multirow[t]{2}{*}{ LOD } & \multirow[t]{2}{*}{ PVE (\%) } \\
\hline & & & & Start (cM) & End (cM) & distance $(\mathrm{cM})$ & & \\
\hline \multirow[t]{5}{*}{${ }^{*} \mathrm{FW}$} & $2018 / 2019$ & qFW10-6.1 & 10 & 65.892 & 71.858 & 5.97 & $4.72-6.01$ & $6.9-8.7$ \\
\hline & 1819 & qFW10-1 & 10 & 17.483 & 34.771 & 17.29 & 6.51 & 9.4 \\
\hline & 1819 & qFW10-2 & 10 & 44.095 & 76.613 & 32.52 & 8.17 & 11.6 \\
\hline & 1819 & qFW6-3 & 6 & 73.682 & 74.556 & 0.87 & 5.4 & 7.8 \\
\hline & 1819 & qFW6-4 & 6 & 87.691 & 88.482 & 0.79 & 5.59 & 8.1 \\
\hline \multirow[t]{5}{*}{${ }^{*} \mathrm{FL}$} & $2018 / 2019$ & qFL10-2.1 & 10 & 44.638 & 56.88 & 12.24 & $6.21-6.54$ & $9-9.5$ \\
\hline & 1819 & qFL10-1 & 10 & 13.739 & 51.909 & 38.17 & 8.88 & 12.4 \\
\hline & 1819 & qFL10-2 & 10 & 57.755 & 58.118 & 0.36 & 5.63 & 8.5 \\
\hline & 1819 & qFL10-3 & 10 & 59.799 & 59.799 & 0 & 5.87 & 8.5 \\
\hline & 1819 & qFL10-4 & 10 & 62.134 & 62.134 & 0 & 5.86 & 8.5 \\
\hline \multirow[t]{3}{*}{ *FD } & 1819 & qFD10-1 & 10 & 4.137 & 39.835 & 35.7 & 9.07 & 12.8 \\
\hline & 1819 & qFD10-2 & 10 & 65.892 & 73.183 & 7.29 & 7.43 & 10.6 \\
\hline & 1819 & qFD10-3 & 10 & 49.704 & 63.503 & 13.8 & 7.02 & 10.1 \\
\hline \multirow[t]{3}{*}{ *FSI } & 1819 & qFSI7-1 & 7 & 104.247 & 108.987 & 4.74 & 5.51 & 8 \\
\hline & 1819 & qFSI7-2 & 7 & 109.885 & 111.761 & 1.88 & 5.45 & 7.9 \\
\hline & 1819 & qFSI8-3 & 8 & 60.56 & 65.763 & 5.2 & 5.89 & 8.5 \\
\hline${ }^{*}$ Nof/ec & 1819 & qNof/ec5-1 & 5 & 67.85 & 69.165 & 1.32 & 5.56 & 8.1 \\
\hline \multirow[t]{2}{*}{${ }^{*}$ Nof/n } & 1819 & qNof/n5-1 & 5 & 61.076 & 92.992 & 31.92 & 8.86 & 12.5 \\
\hline & 1819 & qNof/n9-2 & 9 & 14.055 & 16.446 & 2.390 & 6.71 & 9.6 \\
\hline \multirow[t]{3}{*}{${ }^{*} \mathrm{Nos} / \mathrm{f}$} & 1819 & qNos/f10-1 & 10 & 26.713 & 46.338 & 19.62 & 8.15 & 11.6 \\
\hline & 1819 & qNos/f10-2 & 10 & 48.095 & 48.095 & 0 & 6.22 & 9 \\
\hline & 1819 & qNos/f10-3 & 10 & 50.464 & 50.744 & 0.28 & 5.71 & 8.3 \\
\hline \multirow[t]{7}{*}{${ }^{*} 100 \mathrm{SW}$} & 1819 & q100SW10-1 & 10 & 69.787 & 72.522 & 2.73 & 4.16 & 6.1 \\
\hline & 1819 & q100SW10-2 & 10 & 84.051 & 84.847 & 0.80 & 3.26 & 4.8 \\
\hline & 1819 & q100SW10-3 & 10 & 87.238 & 88.035 & 0.80 & 3.32 & 4.9 \\
\hline & 1819 & q100SW10-4 & 10 & 89.231 & 89.231 & 0 & 3.21 & 4.7 \\
\hline & 1819 & q100SW12-5 & 12 & 30.03 & 30.03 & 0 & 3.03 & 4.5 \\
\hline & 1819 & q100SW4-6 & 4 & 66.739 & 66.739 & 0 & 3.01 & 4.4 \\
\hline & 1819 & q100SW4-7 & 4 & 67.808 & 67.808 & 0 & 3 & 4.4 \\
\hline \multirow[t]{2}{*}{${ }^{*} \mathrm{LL}$} & 2018/2019 & qLL10-2.1 & 10 & 42.768 & 51.909 & 9.14 & $8.71-10.48$ & $12.3-14.6$ \\
\hline & 1819 & qLL10-1 & 10 & 29.322 & 62.784 & 33.46 & 14.21 & 19.3 \\
\hline \multirow[t]{8}{*}{${ }^{*} \mathrm{LD}$} & $2018 / 2019$ & qLD10-2.1 & 10 & 42.768 & 51.909 & 9.14 & $4.3-6.64$ & $6.3-9.5$ \\
\hline & $2018 / 2019$ & qLD12-4.1 & 12 & 27.316 & 32.654 & 5.34 & $3.44-5.91$ & $5.1-8.5$ \\
\hline & 1819 & qLD1-1 & 1 & 3.65 & 12.708 & 9.06 & 6.98 & 10 \\
\hline & 1819 & qLD10-2 & 10 & 34.771 & 37.327 & 2.56 & 6.8 & 9.8 \\
\hline & 1819 & qLD10-3 & 10 & 42.768 & 50.744 & 7.98 & 8.1 & 11.5 \\
\hline & 1819 & qLD12-4 & 12 & 13.421 & 13.421 & 0 & 6.04 & 8.7 \\
\hline & 1819 & qLD12-5 & 12 & 23.146 & 23.146 & 0 & 6.11 & 8.8 \\
\hline & 1819 & qLD12-6 & 12 & 24.122 & 27.342 & 3.22 & 7.17 & 10.3 \\
\hline \multirow[t]{3}{*}{${ }^{*}$ LA } & $2018 / 2019$ & qLA10-2.1 & 10 & 39.835 & 58.118 & 18.28 & $6.46-7.74$ & $9.3-11$ \\
\hline & 1819 & qLA10-1 & 10 & 42.768 & 43.567 & 0.8 & 3.33 & 4.9 \\
\hline & 1819 & qLA10-2 & 10 & 44.095 & 50.744 & 6.65 & 3.71 & 5.4 \\
\hline${ }^{*} \mathrm{FS}$ (SSC) & 2019 & qFS3-1 & 3 & 100.85 & 102.109 & 1.26 & 5.48 & 9.1 \\
\hline
\end{tabular}


Table 5 Promising QTLs detail information detected under 2 individual years and extra year (1819) data (Continued)

\begin{tabular}{|c|c|c|c|c|c|c|c|c|}
\hline \multirow[t]{2}{*}{ Traits } & \multirow[t]{2}{*}{ Year } & \multirow[t]{2}{*}{ QTLS } & \multirow[t]{2}{*}{ LGs } & \multicolumn{3}{|c|}{ Interval (cM) } & \multirow[t]{2}{*}{ LOD } & \multirow[t]{2}{*}{ PVE (\%) } \\
\hline & & & & Start (cM) & End (cM) & distance (cM) & & \\
\hline & 2019 & qFS5-2 & 5 & 10.18 & 10.18 & 0 & 5.4 & 9.1 \\
\hline *FF & 2019 & qFF10-1 & 10 & 74.412 & 76.613 & 1.2 & 4.81 & 8.1 \\
\hline
\end{tabular}

*FW fruit weight, $F L$ fruit length, $F D$ fruit diameter, FS/ fruit shape index, Nof/ec number of fruits/end cluster, Nof/n number of fruits/node, Nos/f number of seeds/ fruit, 100SW 100 seed weight, $L L$ leaf length, LD leaf diameter, LA leaf area, FS (SSC) fruit sweetness (soluble solid contents), FF fruit firmness

spanned an interval ranged from 14.05-100.07 with LOD value between 3.85 to 4.5 and 5.6 to $6.6 \%$ PVE. q100SW7-2 was observed as a major QTL with maximum variance of $6.6 \%$ (Table S3, additional file 6). 7 QTLs targeting 100SW were also found on LG4, 10 and 12 with maximum PVE of $6.1 \%$ for q100SW10-1 in extra year (Table 5).

Leaf length $(l l)$ : 6 QTLs related to leaf length were mapped on LG7, LG10, while promising QTL (qLL102.1) positioned on LG10 starting from 42.77 to 51.91 cM interval bearing highest LOD of 10.48 with explained variance of $14.6 \%$ (Fig. 4). The major QTL (qLL10-2) was detected with maximum 14.6\% PVE. Only 1 QTL ( $q L L 10-1)$ was detected on LG10 with larger PVE of $19.3 \%$ in 1819 (Table 5 and S3, additional file 6).

Leaf diameter $(l d)$ : We observed 14 fruit diameter controlling QTLs were spanned on LG1, 10, 12, among them $q L D 10-2.1$ and $q L D 12-4.1$ represented stability throughout 2 individual years and positioned on LG10 and LG12 with covered distance ranged from 42.77$51.91 \mathrm{cM}$ and 27.32-32.65 cM and corresponding highest LOD of 6.64 and 5.91 and explaining PVE\% of 9.5 and 8.5, respectively (Fig. 4). A $q L D 10-1$ having maximum variance of $11 \%$, considered as major QTL. Another 6 LD QTLs were located on LG1, 10 and 12 with highest PVE of $11.5 \%$ for $q L D 10-3$ in extra year (Table 5 and S3, additional file 6).

Leaf area $(l a)$ : 9 QTLs were found related to leaf area on LG1, 9, 10 and 12. Among them, qLA10-2.1 stable QTL targeting leaf area was detected on LG10 and covered genetic interval ranged from 39.84-58.12 cM with peak LOD of 7.74 and $11 \%$ explained variance (Fig. 4). Moreover, $q L A 10-1$ was regarded as a major QTL based on maximum phenotypic variance of $12.5 \%$. Only 2 QTLs corresponding to LA were found on LG10 with highest PVE of 5.4\% for $q L A 10-2$ in extra year (1819) (Table 5 and S3, additional file 6).

In our results, qLL10-1 was regarded as a major QTL with the largest explained $19.3 \%$ of variance and significant LOD of 14.21 (Table 5). Intriguingly, we encountered an interesting phenomenon of QTL co-localization among fruit and leaf related traits. Specifically, qFL102.1 was overlapped with tightly linked markers corresponding to LL, LD \& LA among stable QTLs and represented a highly significant positive correlation (Tables 1 and 5). The proportion of variance (PVE\%) between promising QTLs was $>10 \%$, which indicated major contribution of stable QTLs controlling corresponding important traits. Moreover, we had noticed a number of co-located QTLs such as fruit weight QTLs shared closely linked map positions with FL, FD, Nos/f, 100SW, LL, LD, LA and FF QTLs on LG10 consistently in 2 individuals year (2018-2019) and extra year (1819), which also indicated highly positive significant correlation as well (Fig. 4, Tables 1 and 5).

\section{Discussion}

SLAF based high-density mapping

A high-density and high-quality genetic map of goji berry assembled in the present study based on $F_{1}$ population derived from 2 different species of Lycium using SLAF-seq strategy, demonstrated to be a highly costeffective technique for the discovery of a large number of SNPs and wide-scale genotyping [34]. SLAF-seq has been utilized in many different crop species, even those without reference genome [33, 42, 46]. Gong et al. (2019) [33], reported very first goji berry high-density genetic map using an intra-specific $F_{1}$ population under ddRAD-seq. While the current perpetual report on genetic mapping using SLAF-seq will further enhance the technology and improve understanding of molecular genetics of goji berry. Though, SLAF-seq approach has rarely been utilized in goji berry to construct a genetic map for QTL analysis. Comparatively, previous GBS based approaches such as RAD and ddRAD-seq have limitation due to technical and procedural complexity, cost unfeasibility and lack of pre-designed scheme and deep sequencing for ensuring accuracy and efficiency [46]. Subsequently, SLAF sequencing takes advantage of high-throughput, cost-effective, deep sequencing technique based on double Barcoding system accompanying a larger population and pre-designed strategy for large scale SNPs, InDel detection and genotyping [34].

In the first place, we contrived a scheme along the foundation of well-assembled reference genome sequence. Due to unavailability of goji genome, we explored goji genetic and DNA content detail [43], and pepper was selected as a reference genome to carry out the sequencing and ensure the uniformity, density and efficiency of markers production. Under the pilot 
experiment, RsaI and HinCII restriction enzymes were chosen and excised fragment (364-414 bp) for sequencing. The SLAF library was evaluated by choosing the rice genome (Oryza sativa L. japonica) as a control for comparing with reference genome to ensure reliability and validity. According to Sun et al. (2013) [34], the recommended quality scores should not be less than Q30 and 6-fold minimal sequencing depth for each individual in SLAF-seq defined pipeline. Our dataset contained 3021.32 Mb paired-end reads and achieved 95.04\% Q30 score. The sequencing depth for parents was 66.43 -fold and 15.23-fold for individuals, which fulfilled the quantity and quality criteria of markers needed to conduct high-density genetic mapping. At last, we abided by strict principles of filtering to get high-quality SLAF markers (See "Methods" section). Two hundred fourteen thousand nine hundred sixty-one polymorphic SLAFs were obtained with a polymorphism rate of $43.47 \%$, higher than previously identified [25, 47, 48]. In the current study, 8 segregation types were received with 24 , 329 maximum number of markers accounting for (aa $\times \mathrm{bb})$ homozygous group, which was larger than previous reports [25, 33]. A total of 5669 high-quality SLAF markers were obtained following filtration process. All the demonstrated outstanding features represented markers accuracy, high-throughput, efficiency at a feasible cost, support further use of the SLAF sequencing technique for goji berry.

\section{High-density genetic map of goji berry}

After building a successful SLAF library and the generation of high-quality markers. Only 3495 SLAFs were integrated properly out of 5669 total SLAF markers in the genetic map along with 15,810 SNPs using High Map software. And spanned final genetic distance of 1649.03 cM on 12 linkage groups (LGs) of goji berry, with an average marker interval of $0.47 \mathrm{cM}$. The linkage map was sufficiently saturated with the smallest mean marker interval of $0.47 \mathrm{cM}$, as compared to previous similar investigations ranged from $0.48-0.95 \mathrm{cM}[29,40,48,49]$. Zhao et al. (2019) [25], reported wolfberry genetic map based on $302 \mathrm{~F}_{1}$ individuals using 6733 SNP markers with total genetic distance of $1702.45 \mathrm{cM}$ and mean interval of $0.31 \mathrm{cM}$. The current linkage map contributed greater potential by accumulating 15,810 SNPs with outstanding attributes than previously reported map [25]. Goji has gained popularity due to economic importance and its historical use as TCM [50]. Thus, molecular breeding and genetics aspects of goji berry still need to be unfolded. In the current study, $305 \mathrm{~F}_{1}$ individuals were selected for high-resolution strategy, though the large number of individuals could bring higher recombination events $[25,51]$, and accelerate the accuracy of fine QTL positioning [52].
The reliability of the genetic map was determined by haplotype maps, heatmaps, maximum gaps, mean gaps $<=5$, integrity of individuals on the map and segregation distortion $(P<0.001)$. The haplotype maps and heat maps generated for each linkage group revealed SLAFs distributed in a regular manner on all 12 LGs and correctly ordered. Moreover, pair-wise recombination rates were found lower significantly among adjacent markers except LG10 and 6. The maximum gap and average gap $<=5$ were larger than previous study [25]. LG1, 4 and 7 were found with varied marker density and maximum gaps greater than $>10 \mathrm{cM}$. Likely, low marker density areas depict strongly homozygous regions of the goji genome, a similar trend observed by Ollitrault et al. (2012) [53]. In contrast, high-density marker areas depict the centromeric location on the chromosomes contributing larger physical distance leading to lesser genetic distance. Relatively, high-density areas may interact with the genome at some position due to interspecific heterozygosity as described by Lindner et al. (2000) [54], Ollitrault et al. (2012) [53]. The integrity of the individuals on the genetic map was $99.03 \%$, which reflected true high-quality and consistency of the map. Meanwhile, only 18 (0.34\%) segregation distortion $(P<0.001)$ markers were observed in LG11 and 12 , significantly lower than previously reported [25, 42]. The segregation distortion among markers might be ascribable to the distant relationship of the parents and gametic or zygotic selection [55].

The current updated high-quality and high-density genetic map were found accurately designed and extremely saturated than earlier studies $[25,41]$. Therefore, we claim that the linkage map is precise and exhibit high-throughput, which would provide insights on the molecular biology of economically important medicinal tree, plant and subsequently strong grounds for QTL mapping and candidate gene identification particularly of fruit size-related traits in goji berry. Further, it will ensure to be an essential source for comparative genomic studies [56], and aid in assembling the reference genome of goji berry.

\section{QTL mapping analysis for fruit size-related traits}

QTL mapping using high-density genetic maps can provide a fruitful estimation in specific quantitative traits and data mining [36]. For conducting QTL analysis, different software is available limited to the crossing models such as IciMapping [57], MapQTL [58], and so on. The current study is based on $F_{1}$ interspecific population and cross-pollinated (CP) model, which is different than other population used for mappings such as RILs, back cross and double haploids [52]. Thus, MapQTL with composite Interval mapping (IM) is definitely a suitable software for conducting QTL analysis 
in CP model, likewise it has been successfully utilized in different species, pepper [52], cucumber [49], and walnut [38]. Zhang et al. (2010) [59], successfully plotted QTL analysis for 8 traits in sweet cherry. In a recent report by Gong et al. (2019) [33], a total of 32 QTLs were found corresponding to six photosynthesis-related traits using MapQTL in L. barbarum L. Similarly, in another study the interspecific high-density genetic map of goji berry has been explored for six agronomically important traits utilizing MapQTL [25].

We employed MapQTL v. 6.0 and characterized a large set of QTLs controlling fruit and leaf-related traits and fruit quality parameters as well. Particularly, 6 promising QTLs observed on LG10 and 12, targeting FW, FL, LL, LD and LA under 2 individual years consistently. For the extra year (1819), 36 QTLs were detected corresponding to FW, FL, FD, FSI, Nof/ec, Nof/n, Nos/f, 100SW, LL, LD and LA (Fig. 4 and S6, Table 5). QTLs detected consistently in 2 years (2018-2019) dataset were regarded as stable QTL. Among several stable QTLs, fruit weight QTL (qFW10-6.1) was detected on LG10 with LOD up to 6.01 and explained phenotypic variance of $8.7 \%$. In contrast, no fruit weight stable QTL was detected in previous study [25]. The fruit weight QTLs have been reported in different Solanaceae species, for example, in tomato $28 \mathrm{FW}$ QTLs were identified. Specifically, fw1.1, fw2.1, fw2.2, fw3.1, fw3.2 and fw11.3 major fruit weight QTLs mapped in almost four interspecific individual studies and accounted for fruit weight variation during tomato evolution [21]. Similarly, 5 fruit weight QTLs were detected in pepper, and fw3.2 was found with significant impact and larger phenotypic variance $\left(R^{2}=0.12-0.15\right)$ [22]. Pereira et al. (2018) [47], investigated QTLs regarding fruit morphological traits in Cucumis melo L. and FWQU5.1 was detected as a major QTL on LG5 controlling fruit weight with maximum PVE of $28.3 \%$. Another study found, 3 QTLs targeting fruit weight in eggplant on different LGs with maximum phenotypic variance of $44 \%$ corresponding to $q F W-9.1$ [12]. For fruit length, we detected promising QTL (qFL10-2.1) on LG10 with peak LOD of 6.54 and 9.5\% phenotypic variance. Zhao et al. (2019) [25] detected 4 stable FL QTLs on LG11 with LOD and PVE up to 17.24 and $30.9 \%$, respectively. Several reports identified fruit length and fruit diameter QTLs spanned on different chromosomes [29, 40,60].

Among the leaf related traits, $q L L 10-2.1$ stable QTL corresponding to leaf length was spanned on LG10, with peak LOD value and PVE up to 10.48 and $14.6 \%$, respectively. Other stable QTLs ( $q L D 10-2.1$ and $q L D 12-$ 4.1) controlling leaf diameter located on LG10 and 12 with the highest LOD of 6.64 and 5.91, and 9.5 and 8.5\% phenotypic variance. The stable QTL responsible for leaf area was $q L A 10-2.1$ spanned on LG10 with corresponding peak LOD of 7.74 and explained variance of $11 \%$.
Nonetheless, previously only two stable QTLs were reported regarding leaf index (qLIIO-2 and qLI11-2) on LG10 and 11, harbored 4 and 20 markers, respectively; while, no stable QTLs controlling leaf length and leaf width were reported [25]. Zhao et al. (2019) [25], detected total 55 QTLs targeting fruit weight, fruit length, fruit index, leaf width and leaf index except leaf length based on more than 2 years phenotypic data. Most of the QTLs were spanned on LG11 indicated cluster region for various traits. Moreover, 18 significant QTLs corresponding to fruit index were detected on LG11 covering an interval of 73.49-90.94 cM and one major QTL (qFL11-3) targeting fruit length was also observed with highest PVE of $30 \%$. Among leaf related traits, 2 stable QTLs (qLI10-2, qLI11-2) targeting leaf index were detected on two different linkage groups for the 3-year dataset. Comparatively, in the present study, we detected 78 QTLs corresponding to all traits under investigation for 2 individual years of dataset along with 36 QTLs observed for an extra year (1819) spanned an interval ranged from $3.65-111.76 \mathrm{cM}$. In addition, 6 stable QTLs (qFW10-3.1, qFL10-2.1, qLL10-2.1, qLD10-2.1, qLD124.1, qLA10-2.1) were observed on LG10 and LG12 targeting fruit size-associated attributes (Fig. 4, Table 5). LG10 was observed loaded with significant QTLs represented as a hot spot region for important fruit and leaf related traits. Interestingly, we found a couple of overlapping QTLs with those detected by Zhao et al. (2019) [25] such as qFSI1-1 and qFI1-2 [25], located both on LG1 and spanned an interval ranged from 123.73$124.76 \mathrm{cM}$ \& 114.81-123.77 cM, respectively. Another QTL set (qNof/ec5-3.1, qNof/n5-1.1 and qLW5) were overlapped on LG5 and covered genetic distance ranged from 68.04-76.61 cM [25] (Table S3, additional file 6). We also encountered colocalization phenomenon of qFL10-2.1 with qLL10-2.1, qLD10-2.1 and qLA10-2.1 along with tightly linked markers among stable QTLs (Fig. 4, S8a-o; Additional file 3). Additionally, it was observed that QTLs corresponding to FW, FL, FD, Nos/f, LL, LD and LA shared similar genetic position on LG10 throughout two individual years as well as in extra year. The co-localization of traits linked QTLs usually reflected positive significant correlation among different traits [22]. For example, fruit diameter and pericarp thickness analysis revealed 3 common positions on the genetic map of pepper $(r=0.86,0.87)$, reflected dual genomic regions with common interaction for both traits [22]. So, we suggest that the current findings could reveal extra potential than previous similar studies and would provide a novel foundation to understand the genetics linked to fruit size-related attributes of goji berry.

It is one assumption that QTLs detected in the same genomic region targeting various traits may occur due to 
pleiotropy or linkage phenomenon [22]. Among promising QTLs, only 17 markers were found closely linked with FL, LL, LD and LA. It is also worth mentioning another set of 5 markers, which were located in stable QTLs related to FL, LL and LA on LG10. In the previous similar study, 3 markers were found significantly related to several traits in pepper, for example, 1 marker (LG8) influenced six traits, another marker (LG2) associated with four traits and third marker (LG3) related with five different fruit development traits [22]. Thus, we may hypothesize that those particular five genomic regions or loci may involve either a specific locus associated with pleiotropy, which might influence different aspects of goji fruit size and plant architecture or several linked loci indicates the specific influence on plant developmental responses. Further studies will require to target these promising QTLs for determining the likely candidate genes affecting different fruit development traits in goji berry. Furthermore, fruit sweetness (SSC) QTLs (qFS3-1, qFS5-2) were detected on LG3 and LG5 with relative LOD of 5.48 and 9.2\% PVE and qFF10-1 responsible for fruit firmness were spanned on LG10 with peak LOD of 4.81 and PVE 8.1\% (Fig. 4, Table 5). Ben Chaim et al. (2001) [22], reported similar events by identifying 3 QTLs in each year, but none as stable, corresponding to soluble solids concentration (SSC) in pepper with the largest phenotypic effect $\left(R^{2}=0.12\right)$. Two QTLs (fi9.1, fi11.1) were detected controlling fruit firmness in pepper with $14 \%$ total variance [22], which indicated close agreement with our findings. Some other study conducted QTL analysis and found 2 QTLs (QBRX2-1, QBrix6) targeting flesh sweetness in watermelon [61].

However, transcriptome sequencing analysis is too indispensable to measure the expression pattern between different fruit and leaf developmental stages, which can exacerbate the mining process of candidate gene identification $[25,49]$. It has been brought out that promising orthologous QTLs or genes in similar crop species influence the same morphological or phenotypic impacts. Some physiologically associated trait comparison has been done practically and identified similar genetic positions [22, 62]. Before the 90s, there was no QTL comparison has been made relating to fruit-producing crops until the identification of fruit associated traits in tomato [21, 63]. Ben Chaim et al. (2001) [22], performed comparative QTL analysis between pepper and tomato and found quite interesting findings regarding fruit shape and fruit weight attributes. The orthologous QTLs in pepper and tomato targeting fruit weight on chromosomes 2 and 3 and one RFLP marker was found closely associated with $f w 2.1$ and $f w 2.2$ in pepper [22], and tomato [64], and another marker with fw3.2 and fw3.1 in pepper [22], and tomato [21], respectively. The above explanation could be helpful in the current study as Goji (Lycium spp.) being relative to the Solanaceae; thus, we might expect similar genetic identities or syntenic association, which existed in tomato and pepper [22, 27], tomato and eggplant [12], and tomato and potato [23], which would be uncovered in later studies. Here, we have utilized common morphological traits related to the fruit and leaf development and these featured traits found to deliver a major contribution for domestication of fruit crops [12, 56]. To further evaluate the potential of goji population and explore additional QTLs, we already have initiated developing recombinant inbred lines (RILs) by selecting full-sib mating with extremely distinguished offspring in 2019. RILs population have been successfully applied in many crop species such as pepper [52], peanut [65], and melon fruit [47]. Moreover, the enhanced QTL analysis could lead to exploring the genetic and molecular characterization of agronomically important fruit traits in goji berry.

\section{Conclusions}

Our findings of goji berry high-resolution linkage map using specific locus amplified fragment sequencing (SLAF-seq), may inculcate a powerful and more precise technique for de novo SNPs detection and high- density genetic map construction for species without reference genome sequence. A total of 3495 high quality SLAF markers was utilized for the construction of genetic map using 305 individuals from the $F_{1}$ interspecific population. The high-density genetic map provides 6 promising QTLs related to fruit weight, fruit length, leaf length, leaf diameter and leaf area. Remarkably, fruit weight, fruit length, fruit diameter, number of seeds per fruits and leaf related traits QTLs were shared similar location on the map, in particular, fruit length QTL (qFL10-2.1) were co-localized corresponding to leaf size-related stable QTLs with tightly linked markers on LG10. We might speculate that these promising QTLs have pleiotropic or linkage relationship controlling several traits. Our results will lay a solid ground for conducting positional cloning and marker-assisted breeding of goji berry. Moreover, our previous report results regarding the high-density genetic map of $L$. barbarum using intra-specific population and the current findings based on interspecific population may prove to be a better source for assembling the goji berry reference genome.

\section{Methods}

\section{Plant materials}

The planting material was comprised of Lycium chinense Mill. cv. "Daye" as the female parent and Lycium barbarum L. cv. "Zhongkelüchuan1 (ZKLC1)" as the male parent. Two cultivated varieties ZKLC1 and Daye were obtained from Goji germplasm reservoirs of Wuhan 
Botanical Garden, Chinese Academy of Sciences, Wuhan and South China Botanical Garden, Chinese Academy of Sciences, Guangzhou, China, respectively. The $\mathrm{F}_{1}$ interspecific pseudo-testcross population was constructed by cross-fertilizing two parents in June 2016 and after sowing in the greenhouse during spring of 2017, the nursery was transplanted in the field at North-West China Bioagricultural Center, $\left(38^{\circ} 28^{\prime} 05^{\prime \prime}\right.$ N, 106 $16^{\prime} 23^{\prime \prime}$ E) Yinchuan City, Ningxia Province, China. The female parent (Daye), also known as Chinese Goji, botanically has ovate to lanceolate leaves, shallow to dark purple flowers, fruit color range from dark red to orange, fruit shape oblong, fruit weight $(0.38 \mathrm{~g})$, fruit length $(1.08 \mathrm{~cm})$, fruit diameter $(0.75 \mathrm{~cm})$ (values are taken from collecting data), and seeds typically broader, rounded than L. barbarum with yellowish to yellow-brown color. The male parent (ZKLC1) has lanceolate to oblong leaves, bright to royal purple flower color, shiny red fruit color, round in shape, fruit weight $(1.05 \mathrm{~g})$, fruit length $(1.79 \mathrm{~cm})$, fruit diameter $(1.28 \mathrm{~cm})$, and seeds semispherical to flattish with light yellow color [66], (The parents Daye and ZKLC1 and progeny plant and fruit pictures can be seen in Fig. 5). In September 2018, 305 individuals randomly selected out of total 450 offspring were used for genetic linkage map construction and QTL mapping analysis, particularly of agronomic importance, fruit size and leaf related morphological traits such as fruit weight (FW), fruit length (FL), fruit diameter (FD), number of fruits per end cluster (Nof/ec), number of fruits per node (Nof/n), number of seeds per fruit (Nos/f), 100 seed weight (100SW), and leaf length (LL), leaf diameter (LD), leaf area (LA). Despite morphological traits, fruit quality traits such as fruit sweetness-soluble solid contents ${ }^{\circ}$ brix FS (SSC) and fruit firmness (FF) were also included based on one-year dataset (2019).

\section{Phenotypic traits estimation}

The morphological trait data were gathered during two crop seasons, the summer (July) of 2018 and 2019. For fruit and leaf related traits: FW, FL and FD, LL, LD and LA, 20 fully ripened fruits and 10 fully grown first true leaves were picked randomly from progeny and parents' plant from the field, then photographed on scale paper $(35 \times 25 \mathrm{~cm})$ by using Nikon D750 DSLR (Nikon Corporation, Japan) in the year 2018. The digital images were processed to measure the above-mentioned traits by ImageJ [48], software v. 1.52a [15, 16]. Conversely, for 2019 summer season, a quicker yet precise method was adopted to assess the fruits and leaves related traits. Firstly, a digital scanner (MICROTEK ScanMaker i600) was employed to photograph the fruits and leaves, then built-in Chinese software Ban Ben, WSEEN ( $v$ 2.1.2.4) used to measure FL, FD, LL, LD and LA according to the developer's instructions. FSI was measured by dividing FL with FD. The other trait data, such as Nof/ec and Nof/ $n$ were collected visually in the field based on a maximum of 10 repeats per individual plant. Nos/f and $100 \mathrm{SW}$ were measured in the laboratory by taking 5 repeats and after overnight complete drying

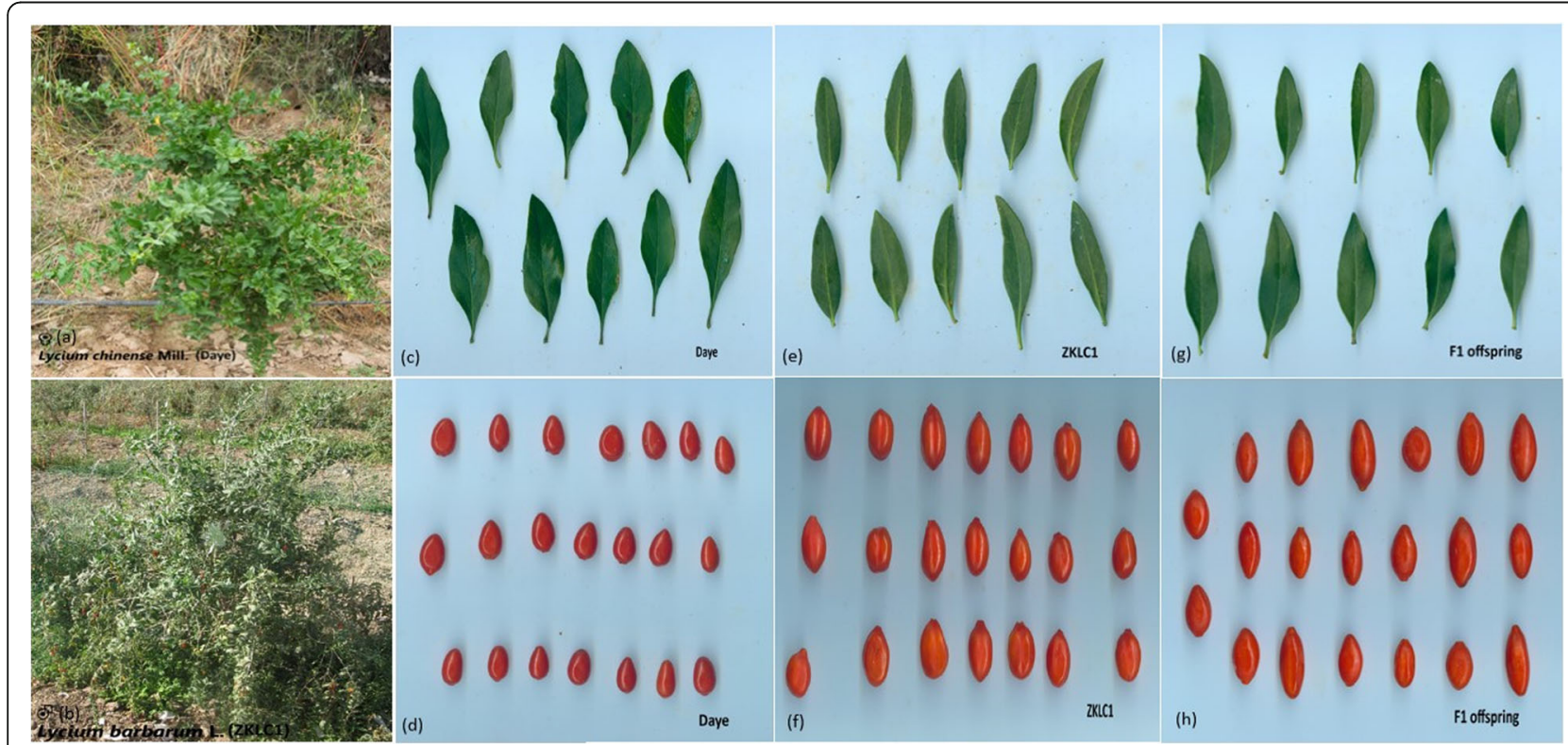

Fig. 5 Female (Lycium chinense Mill. cv. Daye) and male (Lycium barbarum L. CV. ZKLC1) parent plants, and $F_{1}$ offspring pictures. *a female plant, $\mathbf{b}$ male plant, $\mathbf{c}$ female plant leaves, $\mathbf{d}$ female mature fruit, e male plant leaves, $\mathbf{f}$ male mature fruits, $\mathbf{g} \mathrm{F}_{1}$ offspring leaves, $\mathbf{h} \mathrm{F}_{1}$ offspring mature fruits 
100 seeds weighed on a digital balance (Sartorious, BSA224S). For assessing fruit quality traits: FS (SSC) ${ }^{\circ}$ brix, we collected fully ripened fruits from $F_{1}$ population per plant 5 repeats and measured with portable refractometer Pal-1(www.atago.net/), and fruit hardness tester equipment GY-4 (Zhejiang Top Instrument Co., Ltd. Hangzhou, China) were used to estimate FF.

\section{DNA extraction and SLAF library construction and sequencing}

Prior to DNA extraction, the young leaves from parents and 305 progenies were collected and dried with desiccant (silica gel). All the leaves DNA were extracted with Plant Genomic DNA Kit (TIANGEN BIOTECH (BEIJING), CHINA, CO., LTD.) according to the manufacturer's instructions with slight modifications. The quantity and quality of the extracted DNA samples were evaluated with a NanoDrop ND-2000 spectrophotometer (Thermo Fisher Scientific) and electrophoresis with 1\% agarose gel, respectively. Specific length amplified fragment sequencing (SLAF-seq) was performed to genotype the 305 individuals from $F_{1}$ population and two parents according to Sun et al. (2013) [34], with minor modifications. The SLAF-seq strategy framework was adopted as the genomic DNA of each sample was subjected to restriction enzyme digestion using RsaI and HinCII (New England Biolabs, NEB, USA). The excised fragments were integrated with a single nucleotide A using Klenow Fragment $\left(3^{\prime} \rightarrow 5^{\prime}\right.$ exo-, NEB), ligation of dual taglabelled sequencing adapters to the A-tailed fragment was performed with T4 DNA ligase (PAGE purified, Life Technologies, USA) [52]. The PCR amplification was completed by using diluted DNA samples after ligation, Q5 $5^{\circ}$, HF- DNA polymerase (NEB), dNTPs, and primers set (forward primer: 5'-AATGATACGGCGACCA CCGA-3 and reverse primer: 5'-CAAGCAGAAGACGG CATACG-3). The target fragments (364-414 bp) were purified using a QIAquick gel extraction kit (Qiagen, Hilden, Germany). The purification of the PCR products was carried out using Agencourt AMPure XP beads (Beckman Coulter, High Wycombe, UK) and then pooled. The $2 \%$ agarose gel electrophoresis was used for pooled sample separation. After diluting gel-purified products, pair-end sequencing with a standard of $125 \mathrm{bp}$ was performed using an Illumina Hi-Seq 2500 system (Illumina, Inc., San Diego, CA, USA) to sequence the SLAF in the quality-tested library at Biomarker Technologies Corporation, Beijing, China [44]. We used Japanese rice, Oryza sativa L. japonica genome as control with a genome size of $382 \mathrm{Mb}$ (http://rapdb.dna.affrc.go. jp.), which claimed to verify the reliability and validity of the testing process and followed the same treatments in accordance with the goji mapping population.

\section{SLAF sequencing data analysis and segregation pattern grouping}

The grouping and genotyping analysis of SLAF sequencing data was performed in detail as described [34]. The raw reads were identified by sequencing to locate reads of the samples through dual index barcode sequences and low-quality reads of quality score $<20 \mathrm{e}$ were filtered out. The clean reads were clustered together through BLAT software [67], based on the similarity of more than $90 \%$. After trimming of barcodes and the terminal 5-bp positions from each high-quality read, which later defined as one SLAF locus [66]. From each SLAF locus SNP loci were detected among parents, and SLAFs greater than 3 SNPs filtered out. According to the parents and offspring, reads with sequencing depth > 140.28-fold and $>35.80$-fold were used to define alleles corresponding to each SLAF locus, respectively. One SLAF locus can hold mostly 4 genotypes for diploid species, hence more than 4 alleles in SLAF loci considered as repetitive SLAFs and discarded later. SLAFs with 2 to 4 alleles were recognized as polymorphic and known as potential markers. All SLAFs polymorphism loci were clustered into 8 segregation patterns as follows: (ab $\times c d$, ef $\times$ eg, hk $\times$ hk, lm $\times$ ll, nn $\times$ np, aa $\times b b, a b \times c c$, and $c c \times$ ab). As the mapping population was $F_{1}$ interspecific from two highly heterozygous parents, thus all patterns were required to construct a high-density genetic map except (aaxbb).

A Bayesian approach was utilized for genotype scoring and quality as demonstrated [34]. To identify highquality SLAF markers 3 steps were taken into account. Firstly, the markers with average sequencing depths in each offspring found $>35.80$-fold and parents $>140.28$ fold. Secondly, markers exceeding $10 \%$ missing data were discarded. Thirdly, markers with significant segregation distortion based on chi-square test $(P<0.001)$ were disqualified, but added later as additional markers during high-density genetic map construction.

\section{Construction of high-density genetic linkage map and evaluation}

Primarily, the marker loci were segregated into 12 linkage groups (LGs) under modified logarithm of odds (MLOD) scores $>5$. To construct high-quality and highdensity map reliably, an advance high map strategy was required to assemble SLAF markers in a particular sequence and exact genotyping errors within linkage groups [19]. The maximum likelihood method by Van Ooijen [68], was employed to construct a high-density genetic map of goji berry and SMOOTH algorithm was utilized for genotyping error correction [69]. K-nearest neighbor algorithm was used to ascribe missing genotypes [70], and Kosambi mapping description was applied to estimate the genetic map distances between 
adjacent markers in centimorgan (cM) [71]. The genetic map was evaluated further following integrity of genetic maps, haplotype map, heat map and segregation distortion analysis $(P<0.05)$.

\section{QTL mapping analysis}

The QTL mapping analysis was executed for a mean of 11 fruits and leaf size-related traits and 2 fruit qualityrelated attributes and analyzed by MapQTL v. 6.0 [58], which employed inclusive composite interval mapping (ICIM) to detect QTL loci among 12 linkage groups for an integrated map of goji berry. A 1000 permutation test (PT) was performed to determine the logarithm of odds (LOD) for assessing statistical significance of each QTL [72]. For reliability of determining QTLs with major and minor effect, different LOD scores were assumed. At first, a LOD threshold corresponding to 0.99 confidence was considered, and if there was no mapping interval, the threshold value corresponding to 0.95 confidence was used; if there was still no positioning interval, then the LOD threshold value of 0.90 confidence was considered. Lastly, if there was still no mapping interval detected, then the permutation test result was not carried out, and the threshold value was manually lowered to 3.0, 2.5, and 2.0. The phenotype variance percentage as elucidated by its corresponding QTL (phenotypic explained variance \%) was estimated within the segregating population on the basis of population variance using MapQTL. The QTL naming criteria were followed as described by McCouch et al. (1997) [73]. The QTLs positioning and genetic map were assembled by BioMercator v. 4.2.1 [74]., and MapChart v. 2.2 [75].

\section{Statistical analysis}

The general descriptive statistical analysis, including frequency distribution was carried out on Origin Pro v. 2016 (https://www.originlab.com/). The Analysis of variance for 2 individual years and Pearson correlation were accomplished on SPSS v. 25 software package (SPSS Inc., Chicago, IL, United States).

\section{Supplementary information}

Supplementary information accompanies this paper at https://doi.org/10. 1186/s12870-020-02567-1.

Additional file 1: Figure S1(a-z). Frequency distribution histogram of the $305 \mathrm{~F}_{1}$ individuals for all investigated traits based on two individual years (2018-2019), an extra year (1819) and only one year (2019) dataset. Figure S2 The marker integrities of each individual in mapping population. *The $x$-axis indicates all 305 individuals along with the specific code name, while the $y$-axis shows markers integrity.

Additional file 2: Figure S3(a-I). Haplotype mapping of $305 F_{1}$ individuals based on 12 linkage groups of integrated maps. *In each map, horizontal line stands a marker and column shows a chromosome in a sample. The first column of the map shows a paternal chromosome and the second column as maternal chromosome, and individuals are separated by blank columns. Green color indicates first allele from the parent, blue as the second allele from the parent, white color shows not judged event, and grey indicates a missing event. The position where color changes in the same column display reorganization events. Figure S4(a-I). Heatmaps of $305 F_{1}$ individuals based on 12 linkage groups of integrated maps. *Each row and column represent marker arranged in the orderliness of the map. Every small square show recombination rate between two markers. The color change trend from yellow to red to purple display reorganization rate from small to large. Yellow color indicates closer marker recombination rate, whereas closer the color from yellow to purple farther becomes the recombination rate.

Additional file 3: Figure $\mathbf{S 8}(\mathbf{a}-\mathbf{o})$. Stable QTLs map position on linkage group 10 (LG10) in 2 individual years (2018-2019) and extra year (1819). *left $>$ 2018, center $>$ 2019, right > 1819, FW_fruit weight, FL_fruit length, LL_leaf length, LD_leaf diameter, LA_leaf area. The red line shows phenotypic explained variation (Expl\%), blue line LOD value, grey line LOD threshold value.

Additional file 4: Table S1. Combined analysis of variance (ANOVA) among 2 individual years (2018-2019) and 11 phenotypic traits data collected from 305 offspring of F1.

Additional file 5: Table S2. Descriptive statistics among 13 attributes based on 2 individual year (2018-2019) and extra year (1819) dataset.

Additional file 6: Table S3. All QTLs detected during 2 indivdual years 2018-2019.

Additional file 7: Figure S5. QTL mapping on integrated linkage map in 2018. *The different color pattern represents identified QTLs for agronomic traits of goji berry such as FW_fruit weight, FL_fruit length, FSI_fruit shape index, Nof/ec_ number of fruits per end cluster, Nof/n_

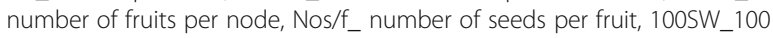
seed weight, LL_leaf length, LD_leaf diameter, LA_leaf area.

Additional file 8: Figure S6. QTL mapping on integrated linkage map in 2019. *The different color pattern represents identified QTLs for agronomic traits of goji berry such as FW_fruit weight, FL_fruit length, FSI_fruit shape index, Nof/ec_ number of fruits per end cluster, Nof/n_ number of fruits per node, Nos/f_ number of seeds per fruit, 100SW_100 seed weight, LL_leaf length, LD_leaf diameter, LA_leaf area, FS_fruit sweetness ( ${ }^{\circ}$ brix) and FF_ fruit firmness

Additional file 9: Figure S7. QTL mapping on integrated linkage map in 1819. *The different color pattern represents identified QTLs for agronomic traits of goji berry such as FW_fruit weight, FL_fruit length, FSI_fruit shape index, Nof/ec_ number of fruits per end cluster, Nof/n_ number of fruits per node, Nos/f_ number of seeds per fruit, 100SW_100 seed weight, LL_leaf length, LD_leaf diameter, LA_leaf area.

\section{Abbreviations}

SLAF-seq: Specific length amplified fragments-sequencing; SNP: Single nucleotide polymorphism; CM: Centimorgan; ZKLC1: Zhongkelüchuan1; cv: Cultivar; LOD: Logarithm of odds; QTL: Quantitative Trait Loci; LG: Linkage group; MLOD: Modified Logarithm of odds; SSC: Soluble solid contents; PVE: Phenotypic variance explained; InDel: Insertion/Deletion;

GBS: Genotyping by sequencing; ddRAD: Double digest restriction-site associated DNA; $F_{1}$ : First filial; MAS: Marker-assisted selection; ANOVA: Analysis of variance; ICIM: Inclusive composite interval mapping; NGS: Next generation sequencing; BLAT: BLAST like alignment tool

\section{Acknowledgements}

The author is greatly thankful to the Chinese Academy of Sciences and The World Academy of Sciences (CAS-TWAS) for awarding PhD President's Fellowship. We would like to express our gratitude to Ms. Junjun Yang, Mr. Guanshen Liu and Mr. Xiaowei Zhou of the Biomarker Technologies Corporation, Beijing, for their technical and analytical assistance. We also thank three anonymous reviewers and editor-in-chief for constructive comments and suggestions to further improve the quality and discernment of the manuscript.

\section{Authors' contributions}

Experiment designed, conceived and supervised HH, YW, FR, Population developed HG, FR, PA, Population maintenance and field management TY, 
LZ, LP, Phenotype data collected and analyzed FR, QTL mapping analysis FR, HG, Manuscript drafted FR, Critically reviewed and constructive suggestions HH, YW, SZ, Revised manuscript and language edited FR, YW. All authors read and approved for final submission.

\section{Funding}

This work was supported by grants from Strategic and Priority Research Program of Chinese Academy of Sciences [XDA24030502, XDA13020604], National Key R\&D Program of China [2018YFD1000607]; the National Natural Science Foundation of China [31770334]; Youth Innovation Promotion Association CAS [2015286]; Science and Technology Program of Guangzhou [201904010167]. The funding organizations did not aid in designing, data collection, analysis, data interpretation and manuscript write up.

\section{Availability of data and materials}

The raw SLAF-sequencing data reported in this paper have been deposited in the Genome Sequence Archive [76], in National Genomics Data Center [77], Beijing Institute of Genomics (China National Center for Bioinformation), Chinese Academy of Sciences, under accession number(s) CRA002920 that are publicly accessible at https://bigd.big.ac.cn/gsa. The remaining datasets used to support the conclusions of this article are included within the article and its additional files.

\section{Ethics approval and consent to participate}

Not applicable.

\section{Consent for publication}

Not applicable.

\section{Competing interests}

All authors declare no competing financial interest.

\section{Author details}

'Key Laboratory of South China Agricultural Plant Molecular Analysis and Genetic Improvement, Guangdong Provincial Key Laboratory of Applied Botany, South China Botanical Garden, Chinese Academy of Sciences, Guangzhou 510650, China. ${ }^{2}$ University of Chinese Academy of Sciences, Beijing 100049, China. ${ }^{3}$ Center of Economic Botany, Core Botanical Gardens, Chinese Academy of Sciences, Guangzhou 510650, China. ${ }^{4}$ Bairuiyuan Company, Yinchuan 750000, Ningxia, China. ${ }^{5}$ GNNU-SCBG Joint Laboratory of Modern Agricultural Technology, College of Life Sciences, Gannan Normal University, Ganzhou 341000, Jiangxi, China.

\section{Received: 3 February 2020 Accepted: 22 July 2020}

\section{Published online: 15 October 2020}

\section{References}

1. Amagase H, Farnsworth NR. A review of botanical characteristics, phytochemistry, clinical relevance in efficacy and safety of Lycium barbarum fruit (goji). Food Res Int. 2011;44(7):1702-17.

2. Zhao Q, Dong B, Chen J, Zhao B, Wang X, Wang L, Wang Y. Effect of drying methods on physicochemical properties and antioxidant activities of wolfberry (Lycium barbarum) polysaccharide. Carbohydr Polym. 2015;127: 176-81

3. Wang CC, Chang SC, Inbaraj BS, Chen BH. Isolation of carotenoids, flavonoids and polysaccharides from Lycium barbarum $L$. and evaluation of the antioxidant activity. Food Chem. 2010;120:184-92.

4. Zhang M, Chen $\mathrm{H}$, Huang J, Li Z, Zhu C, Zhang S. Effect of lycium barbarum polysaccharide on human hepatoma QGY7703 cells: inhibition of proliferation and induction of apoptosis. Life Sci. 2005;76:2115-24.

5. Zhang M, Tang X, Wang F, Zhang Q, Zhang Z. Characterization of Lycium barbarum polysaccharide and its effect on human hepatoma cells. Int J Biol Macromol. 2013;61:270-5.

6. Mocan A, Vlase L, Vodnar DC, Bischin C, Hanganu D, Gheldiu AM, Crișan G. Polyphenolic content, antioxidant and antimicrobial activities of Lycium barbarum L. and Lycium chinense mill. Leaves. Molecules. 2014;19(7):1005673.

7. Yao R, Heinrich M, Zou Y, Reich E, Zhang X, Chen Y, Weckerle CS. Quality variation of Goji (fruits of Lycium spp.) in China: a comparative morphological and metabolomic analysis. Front Pharmacol. 2018;9:151.
8. $\quad$ Tingting $S$, Xiaobo Z, Jiyong $S$, Zhihua $L$, Xiaowei $H$, Yiwei $X$, Wu C. Determination geographical origin and wolfberry of goji berry using nearinfrared spectroscopy and chemometrics. Food Anal Methods. 2016;9:68-79.

9. Lemmon ZH, Reem NT, Dalrymple J, Soyk S, Swartwood KE, Rodriguez-Leal D, Lippman ZB. Rapid improvement of domestication traits in an orphan crop by genome editing. Nat Plants. 2018;4(10):766-70.

10. Frary A, Nesbitt TC, Frary A, Grandillo S, Van Der Knaap E, Cong B, Tanksley SD. fw2.2: a quantitative trait locus key to the evolution of tomato fruit size. Science. 2000;289(5476):85-8.

11. Cong B, Barrero LS, Tanksley SD. Regulatory change in YABBY-like transcription factor led to evolution of extreme fruit size during tomato domestication. Nat Genet. 2008:40(6):800.

12. Doganlar S, Frary A, Daunay MC, Lester RN, Tanksley SD. A comparative genetic linkage map of eggplant (Solanum melongena) and its implications for genome evolution in the Solanaceae. Genet. 2002a;161:1697-711.

13. Guo M, Rupe MA, Dieter JA, Zou J, Spielbauer D, Duncan KE, Simmons CR. Cell number Regulator1 affects plant and organ size in maize: implications for crop yield enhancement and heterosis. Plant Cell. 2010;22(4):1057-73.

14. Hernández-Bautista A, Lobato-Ortiz R, Cruz-Izquierdo S, García-Zavala JJ, Chávez-Servia JL, Hernández-Leal E, Bonilla-Barrientos O. Fruit size QTLs affect in a major proportion the yield in tomato. Chil J Agric Res. 2015;75(4):402-9.

15. Chakrabarti M, Zhang NA, Sauvage C, Muños S, Blanca J, Cañizares J, Causse M. A cytochrome $\mathrm{P} 450$ regulates a domestication trait in cultivated tomato. Proc Natl Acad Sci. 2013;110(42):17125-30.

16. Mu QI, Huang Z, Chakrabarti M, Illa-Berenguer E, Liu X, Wang Y, van der Knaap E. Fruit weight is controlled by cell size regulator encoding a novel protein that is expressed in maturing tomato fruits. PLoS Genet. 2017;13(8): e1006930.

17. Butler L. New linkage groups in the tomato. J Hered. 1951;42:100-4.

18. Rodríguez-Leal D, Lemmon ZH, Man J, Bartlett ME, Lippman ZB. Engineering quantitative trait variation for crop improvement by genome editing. Cell. 2017:171(2):470-80.

19. Liu D, Ma C, Hong W. Construction and analysis of high-density linkage map using high-throughput sequencing data. PLoS One. 2014:9:e98855.

20. Runions A, Tsiantis M, Prusinkiewicz P. A common developmental program can produce diverse leaf shapes. New Phytol. 2017;216:401-18.

21. Grandillo S, Ku HM, Tanksley SD. Identifying the loci responsible for natural variation in fruit size and shape in tomato. Theor Appl Genet. 1999;99(6): 978-87.

22. Ben Chaim A, Paran I, Grube RC, Jahn M, Van Wijk R, Peleman J. QTL mapping of fruit-related traits in pepper (Capsicum annuum). Theor Appl Genet. 2001;102(6-7):1016-28.

23. Tanksley SD, Ganal MW, Prince JP, De Vicente MC, Bonierbale MW, Broun P, Martin GB. High density molecular linkage maps of the tomato and potato genomes. Genetics. 1992;132(4):1141-60.

24. Chen J, Chao CT, Wei X. Goji berry breeding: current status and future prospects. In: Breeding and Health Benefits of Fruit and Nut Crops. London: IntechOpen; 2018. https://doi.org/10.5772/intechopen.76388.

25. Zhao J, Xu Y, Li H, Yin Y, An W, Li Y, Wang Y, Fan Y, Wan R, Guo X, Cao Y. A SNP-based high-density genetic map of leaf and fruit related quantitative trait loci in wolfberry (Lycium Linn.). Front Plant Sci. 2019:10:977.

26. Lin T, Zhu G, Zhang J, Xu X, Yu Q, Zheng Z, Huang Z. Genomic analyses provide insights into the history of tomato breeding. Nat Genet. 2014; 46(11):1220-6.

27. Zygier S, Chaim AB, Efrati A, Kaluzky G, Borovsky Y, Paran I. QTLs mapping for fruit size and shape in chromosomes 2 and 4 in pepper and a comparison of the pepper QTL map with that of tomato. Theor Appl Genet. 2005;111(3):437-45.

28. Zhao X, Huang L, Zhang X, Wang J, Yan D, Li J, Shi T. Construction of highdensity genetic linkage map and identification of flowering-time QTLs in orchardgrass using SSRs and SLAF-seq. Sci Rep. 2016;6:29345.

29. Zhu WY, Huang L, Chen L, Yang JT, Wu JN, Qu ML, Pan JS. A high-density genetic linkage map for cucumber (cucumis sativus I.): based on specific length amplified fragment (slaf) sequencing and QTL analysis of fruit traits in cucumber. Front Plant Sci. 2016;7:437.

30. Carlier JD, Reies A, Duval MF, Coppens-D'Eeckenbrugge G, Leitao JM. Genetic maps of RAPD, AFLP and ISSR markers in Ananas bracteatus and A. comosus using the pseudo-testcross strategy. Plant Breed. 2004;123:186-92.

31. Ritter E, Gebhardt C, Salamini F. Estimation of recombination frequencies and construction of RFLP linkage maps in plants from crosses between heterozygous parents. Genet. 1990;125:645-54. 
32. Ritter E, Salamini F. The calculation of recombination frequencies in crosses of allogamous plant species with applications to linkage mapping. Genet Res. 1996;67:55-65.

33. Gong H, Rehman F, Yang T, Li Z, Zeng S, Pan L, Wang Y. Construction of the first high-density genetic map and QTL mapping for photosynthetic traits in Lycium barbarum L. Mol Breed. 2019;39(7):106.

34. Sun X, Liu D, Zhang X, Li W, Liu H, Hong W, Xu C. SLAF-seq: an efficient method of large-scale de novo SNP discovery and genotyping using highthroughput sequencing. PLoS One. 2013;8:e58700.

35. Pootakham W, Ruang-Areerate P, Jomchai N, Sonthirod C, Sangsrakru D, Yoocha T, Tangphatsornruang S. Construction of a high-density integrated genetic linkage map of rubber tree (Hevea brasiliensis) using genotyping-bysequencing (GBS). Front Plant Sci. 2015;6:367.

36. Zhang L, Guo D, Guo L, Guo Q, Wang H, Hou X. Construction of a highdensity genetic map and QTLs mapping with GBS from the interspecific F1 population of $P$. ostii 'Fengdan Bai'and P. suffruticosa 'Xin Riyuejin'. Sci Hortic. 2019;246:190-200.

37. Liu C, Zhou Q, Dong L, Wang H, Liu F, Weng J, Xie C. Genetic architecture of the maize kernel row number revealed by combining QTL mapping using a high-density genetic map and bulked segregant RNA sequencing. BMC Genomics. 2016;17:915.

38. Zhu Y, Yin Y, Yang K, Li J, Sang Y, Huang L, Fan S. Construction of a highdensity genetic map using specific length amplified fragment markers and identification of a quantitative trait locus for anthracnose resistance in walnut (Juglans regia L.). BMC Genomics. 2015;16(1):614.

39. Zhao Z, Gu H, Sheng X, Yu H, Wang J, Huang L, Wang D. Genome-wide single-nucleotide polymorphisms discovery and high-density genetic map construction in cauliflower using specific-locus amplified fragment sequencing. Front Plant Sci. 2016;7:334.

40. Wei Q, Wang W, Hu T, Hu H, Wang J, Bao C. Construction of a SNP-based genetic map using SLAF-Seq and QTL analysis of morphological traits in eggplant. Front Genet. 2020;11:178.

41. Liu T, Guo L, Pan Y, Zhao Q, Wang J, Song Z. Construction of the first highdensity genetic linkage map of Salvia miltiorrhiza using specific length amplified fragment (SLAF) sequencing. Sci Rep. 2016;6:24070.

42. Luo C, Shu B, Yao Q, Wu H, Xu W, Wang S. Construction of a high-density genetic map based on large-scale marker development in mango using specific-locus amplified fragment sequencing (SLAF-seq). Front Plant Sci. 2016;7:1310.

43. Chen J, Liu X, Zhu L, Wang Y. Nuclear genome size estimation and karyotype analysis of Lycium species (Solanaceae). Sci Hortic. 2013;151:46-50.

44. Biomarker Technologies Corporation, Beijing, China. http://www.biomarker. com.cn/english/.

45. Zhang J, Zhang Q, Cheng T, Yang W, Pan H, Zhong J, Liu E. High-density genetic map construction and identification of a locus controlling weeping trait in an ornamental woody plant (Prunus mume Sieb. Et Zucc). DNA Res. 2015;22(3):183-91.

46. Peterson BK, Weber JN, Kay EH, Fisher HS, Hoekstra HE. Double digest RADseq: an inexpensive method for de novo SNP discovery and genotyping in model and non-model species. PLoS One. 2012;7(5):e37135.

47. Pereira L, Ruggieri V, Pérez S, Alexiou KG, Fernández M, Jahrmann T, GarciaMas J. QTL mapping of melon fruit quality traits using a high-density GBSbased genetic map. BMC Plant Biol. 2018;18(1):324

48. Schneider CA, Rasband WS, Eliceiri KW. NIH image to ImageJ: 25 years of image analysis. Nat Methods. 2012;9:671-5.

49. Wei QZ, Wang Y, Qin X, Zhang Y, Zhang Z, Wang J, Chen J. An SNP-based saturated genetic map and QTL analysis of fruit-related traits in cucumber using specific-length amplified fragment (SLAF) sequencing. BMC Genomics. 2014;15(1):1158.

50. Upton R, Garran TA, Casper L, Laenger R, Patrone C, Reich E, Karioti A, Bergonzi MC, Vincieri FF, Bilia AR, Gardner Z, Chen J. Lycium (Goji) Berry. In: American Herbal Pharmacopoeia and Therapeutic Scotts Valley: 2019.

51. De Massy B. Initiation of meiotic recombination: how and where? Conservation and specificities among eukaryotes. Annu Rev Genet. 2013;47:563-99.

52. Zhang XF, Wang GY, Dong TT, Chen B, Du HS LCB, Geng SS. High-density genetic map construction and QTL mapping of first flower node in pepper (Capsicum annuum L.). BMC Plant Biol. 2019;19(1):167.

53. Ollitrault P, Terol J, Chen C, Federici CT, Lotfy S, Hippolyte I, Costantino G. A reference genetic map of $C$. clementina hort. ex Tan.; citrus evolution inferences from comparative mapping. BMC Genomics. 2012;13:593.
54. Lindner KR, Seeb JE, Habicht C, Knudsen KL, Kretschmer E, Reedy DJ, Allendorf FW. Gene-centromere mapping of 312 loci in pink salmon by halftetrad analysis. Genome. 2000;43(3):538-49.

55. Wang C, Zhu C, Zhai H, Wan J. Mapping segregation distortion loci and quantitative trait loci for spikelet sterility in rice (Oryza sativa L.). Genet Res. 2005;86(2):97-106

56. Van der Knaap E, Chakrabarti M, Chu YH, Clevenger JP, Illa-Berenguer E, Huang Z, Wu S. What lies beyond the eye: the molecular mechanisms regulating tomato fruit weight and shape. Front Plant Sci. 2014;5:227.

57. Meng L, Li H, Zhang L, Wang J. QTL IciMapping: integrated software for genetic linkage map construction and quantitative trait locus mapping in biparental populations. Crop J. 2015;3:269-83.

58. Ooijen V. MapQTL ${ }^{\otimes} 6$, Software for the mapping of quantitative trait loci in experimental populations. Wageningen: Kyazma B.V; 2004.

59. Zhang G, Sebolt AM, Sooriyapathirana SS, Wang D, Bink MC, Olmstead JW, lezzoni AF. Fruit size QTL analysis of an F1 population derived from a cross between a domesticated sweet cherry cultivar and a wild forest sweet cherry. Tree Genet Genomes. 2010;6(1):25-36.

60. Weng Y, Colle M, Wang Y, Yang L, Rubinstein M, Sherman A, Grumet R. QTL mapping in multiple populations and development stages reveals dynamic quantitative trait loci for fruit size in cucumbers of different market classes. Theor Appl Genet. 2015;128:1747-63.

61. Ren Y, McGregor C, Zhang Y, Gong G, Zhang H, Guo S, Xu Y. An integrated genetic map based on four mapping populations and quantitative trait loci associated with economically important traits in watermelon (Citrullus lanatus). BMC Plant Biol. 2014;14:33.

62. Lee M. Comparative genetic and QTL mapping in sorghum and maize. Symp Soc Exp Biol. 1996;50:31-8.

63. Bernacchi D, Beck-Bunn T, Eshed Y, Lopez J, Petiard V, Uhlig J, Tanksley S. Advanced backcross QTL analysis in tomato. I. Identification of QTL for traits of agronomic importance from Lycopersicon hirsutum. Theor Appl Genet. 1998;97:381-97.

64. Alpert KB, Tanksley SD. High-resolution mapping and isolation of a yeast artificial chromosome contig containing fw2.2: a major fruit weight quantitative trait locus in tomato. Proc Natl Acad Sci U S A. 1996;93:15503-7.

65. Li L, Yang X, Cui S, Mu G, Hou M, He M, Liu L. Construction of High-Density Genetic Map and Mapping Quantitative Trait Loci for Growth Habit-Related Traits of Peanut (Arachis hypogaea L.). Front Plant Sci. 2019;10:745.

66. Tatusov RL, Galperin MY, Natale DA, Koonin EV. The COG database: a tool for genome-scale analysis of protein functions and evolution. Nucleic Acids Res. 2000;28(1):33-6.

67. Kent WJ. BLAT-the BLAST-like alignment tool. Genome Res. 2002;12(4): 656-64.

68. Van Ooijen J. Multipoint maximum likelihood mapping in a full-sib family of an outbreeding species. Genet Res. 2011;93:343-9.

69. Van Os H, Stam P, Visser RG, van Eck HJ. SMOOTH: a statistical method for successful removal of genotyping errors from high-density genetic linkage data. Theor Appl Genet. 2005;112:187-94.

70. Huang X, Zhao Y, Li C, Wang A, Zhao Q, Li W, Lu Y. Genome-wide association study of flowering time and grain yield traits in a worldwide collection of germplasm. Nat Genet. 2012;44:32-9.

71. Kosambi $D$. The estimation of map distances from recombination values. Ann Eugenics. 1943;12:172-5.

72. Churchill GA, Doerge RW. Empirical threshold values for quantitative trait mapping. Genet. 1994;138(3):963-71.

73. McCouch SR, Cho YG, Yano M, Paul E, Blinstrub M. Report on QTL nomenclature. Rice Genet Newslett. 1997;14:11-3.

74. Arcade A, Labourdette A, Falque M, Mangin B, Chardon F, Charcosset A, Joets J. BioMercator: integrating genetic maps and QTL towards discovery of candidate genes. Bioinformatics. 2004;20(14):2324-6.

75. Voorrips R. MapChart: software for the graphical presentation of linkage maps and QTLs. J Hered. 2002;93:77-8.

76. GSA. Genome Sequence Archive. Genomics Proteomics Bioinformatics. 2017:15:14-8 [PMID=28387199].

77. National Genomics Data Center M, Partners. Database resources of the National Genomics Data Center in 2020. Nucleic Acids Res. 2020:48(D1): D24-33.

\section{Publisher's Note}

Springer Nature remains neutral with regard to jurisdictional claims in published maps and institutional affiliations. 